\title{
Computational-design to reduce conformational flexibility and aggregation rates of an antibody Fab fragment
}

Cheng Zhang, Maariyah Samad, Haoran Yu, Nesrine Chakroun, David Hilton, Paul

$$
\text { A. Dalby* }
$$

Department of Biochemical Engineering, University College London, Gordon Street, London, WC1E 7JE, UK

*Correspondence email: p.dalby@ucl.ac.uk 


\section{ABSTRACT}

Computationally-guided semi-rational design has significant potential for improving the aggregation kinetics of protein biopharmaceuticals. While improvement in the global conformational stability can stabilise proteins to aggregation under some conditions, previous studies suggest that such an approach is limited because thermal transition temperatures $\left(T_{\mathrm{m}}\right)$ and the fraction of protein unfolded $\left(f_{\mathrm{T}}\right)$ tend to only correlate with aggregation kinetics where the protein is incubated at temperatures approaching the $T_{\mathrm{m}}$. This is because under these conditions, aggregation from globally unfolded protein becomes dominant. However, under native conditions, the aggregation kinetics are presumed to be dependent on local structural fluctuations or partial unfolding of the native state, that reveal regions of high propensity to form protein-protein interactions that lead to aggregation.

In this work, we have targeted the design of stabilising mutations to regions of the A33 Fab surface structure, that were predicted to be more flexible. This Fab already has high global stability, and global unfolding is not the main cause of aggregation under most conditions. Therefore, the aim was to reduce the conformational flexibility and entropy of the native protein at various locations, and thus identify which of those regions has the greatest influence on the aggregation kinetics.

Highly dynamic regions of structure were identified through both molecular dynamics simulation, and B-factor analysis of related X-ray crystal structures. The most flexible residues were mutated into more stable variants, as predicted by Rosetta, which evaluates the $\Delta \Delta G_{\mathrm{ND}}$ for each potential point mutation. Additional destabilising 
variants were prepared as controls to evaluate the prediction accuracy, and also to assess the general influence of conformational stability on aggregation kinetics.

The thermal conformational stability, and aggregation rates of eighteen variants at $65^{\circ} \mathrm{C}$, were each examined at $\mathrm{pH} 4,200 \mathrm{mM}$ ionic strength, under which conditions the initial wild-type protein was $<5 \%$ unfolded. Variants with decreased $T_{\mathrm{m}}$ values led to more rapid aggregation due to an increase in the fraction of protein unfolded under the conditions studied. As expected, no significant improvements were observed in the global conformational stability as measured by $T_{\mathrm{m}}$. However, six of the twelve stable variants led to an increase in the cooperativity of unfolding, consistent with lower conformational flexibility and entropy in the native ensemble. Three of these had 5$11 \%$ lower aggregation rates, and their structural clustering indicated that the local dynamics of the $\mathrm{C}$-terminus of the heavy chain had a role in influencing the aggregation rate.

Keywords: Fab, mutagenesis, aggregation, thermal stability, melting temperature $\left(T_{\mathrm{m}}\right)$, global unfolding, molecular dynamics, protein engineering, cooperativity, entropy

\section{INTRODUCTION}

The aggregation of therapeutic proteins is a prevailing challenge for the formulation of final dosage biopharmaceuticals ${ }^{1}$. It occurs when suboptimal conditions are used for manufacture $^{2}$, transportation ${ }^{3}$ and storage ${ }^{4}$. Typically, certain protein drugs, like 
antibodies, need to be injected in high-concentration dosage forms, and in small volumes for their ease of administration, which leads to an increased risk of aggregation and shear stress 5,6 Aggregation of protein drugs cannot only reduce their efficacy, but can also lead to unwanted adverse immunogenicity in patients ${ }^{7,8}$.

When striving for an optimal storage formulation that forms less than $1 \%$ aggregate over one year, measuring the rates of monomer loss for a wide range of potential formulations and variants, would require many samples, and also a timescale of many months, that is often not practical. While formulation through excipients and solution conditions (e.g. $\mathrm{pH}$, osmolarity, ionic strength) remain as the primary methods to stabilise protein drug candidates, modification of the protein structure for improved stability is also a promising strategy, provided the efficacy of the drug is not compromised $^{9}$. Therefore, in silico screening is emerging as a potential method for predicting the behaviour of mutational candidates ${ }^{10,11}$. The advance of computational molecular dynamics (MD) simulations, with a range of available forcefields ${ }^{12-16}$ has enabled the modelling of complex biomolecular dynamics and interactions, and can be used to evaluate the basis upon which mutations lead to increased conformational stability $^{17}$. Calculation of the root mean square fluctuation (RMSF), measures the deviation over time between an atom or residue position and its reference position. This has been shown to increase significantly in MD simulations at residues around mutations that increase conformational flexibility ${ }^{18}$. RMSF can also be used to guide mutagenesis, with the aim of replacing highly flexible residues with amino acids that lead to more stability through improved interactions or packing ${ }^{19}$. Similarly, B-factors 
(or temperature factors) determined by X-ray crystallography, represent the extent of thermal motion of an atom such that a high B-factor value indicates a high fluctuation for that atom ${ }^{20}$. Thus B-factors have also been used to guide iterative saturation mutagenesis and increase the thermostability of enzymes, by modifying only sequence regions with the highest B-factors ${ }^{21}$. A recent study combined both RMSF and B-factor analyses to identify the flexible loops of transketolase, and engineered more thermostable variants with increased specific activity ${ }^{22}$.

A range of computational methods have also been developed to predict the change in protein stability $\left(\Delta \Delta G_{\mathrm{ND}}\right)$ upon mutation, though most do not yield $\mathrm{R}^{2}$ correlations to experimental $\Delta \Delta G_{\mathrm{ND}}$ of more than $0.5-0.6^{23-25}$. However, Rosetta has achieved considerable progress in structure prediction ${ }^{26}, \operatorname{design}^{27}$, stability improvement, and protein molecule docking ${ }^{28}$. A thorough optimisation of relaxation approaches upon point mutation, found that a minimisation method involving limited backbone minimisation after repacking of all sidechains, achieved the highest experimentprediction correlation coefficient of $0.69^{29}$. Mutations predicted by Rosetta to be conformationally stabilising have been shown previously to enable reductions in the aggregation rates of proteins under partially unfolding conditions ${ }^{30}$.

While monoclonal antibodies $(\mathrm{mAb})$ remained as the leading novel therapeutics for the past a few decades ${ }^{31}$, the complexity (e.g. Fc region) and heterogeneity of mAb (glycosylation, charge variants) make it a challenge for expression ${ }^{32}$ and formulation ${ }^{33}$, compared to antibody fragments like single-chains or single-domains. Antigen-binding fragment (Fab) is one of the widely studied antibody subclasses, with one light chain 
disulphide-linked with one constant heavy chain (CH1) and variable heavy chain $(\mathrm{VH})$ of the heavy chain of a full antibody ${ }^{34}$. Understanding the stability and aggregation propensity of Fab would not only help the discovery of new therapeutic variants, but will also provide valuable insights as a building block for other novel Fab formats like bispecifics $\left(\mathrm{Fab}_{2}\right)$ and trispecifics $\left(\mathrm{Fab}_{3}\right)^{35}$.

Previously, the A33 Fab was studied across a wide range of $\mathrm{pH}$, ionic strength and temperature $^{36}$. It was found that the aggregation rates could only be correlated well with thermal transition temperatures $\left(T_{\mathrm{m}}\right)$ at an elevated incubation temperature of $65{ }^{\circ} \mathrm{C}$, while the correlations dropped substantially at $4-45^{\circ} \mathrm{C}$, consistent with previous observations on IgG variants ${ }^{37}$. The elevated temperature increased the fraction of globally unfolded protein $f_{\mathrm{T}}>0.01$, and led to conditions in which the observed aggregation was dominated by a global unfolding mechanism. However, some conditions at $65^{\circ} \mathrm{C}$ and also all conditions at the lower temperatures relevant to longterm storage, had $f_{\mathrm{T}}<0.01$, where increasing the $T_{\mathrm{m}}$ or lowering $f_{65}$, did not decrease the aggregation rate. Thus, under native conditions at lower temperatures, colloidal stabilisation, local structure dynamics, and protein surface properties within the nativestate ensemble, played more dominant roles in aggregation.

It is known that colloidal stability and conformational stability are two key factors that influence the rate and extent of aggregation ${ }^{38}$. Conformational stability can be separated into the effects of both global and local unfolding, with the latter leading to an increased range of conformations in the native-state ensemble, of which some may be more prone to aggregation than others. It is difficult to predict which specific local 
fluctuations in structure would contribute most to aggregation. We therefore introduced twelve stabilising, and five destabilising mutations, mostly into the regions of A33Fab predicted to be the most flexible. As the Fab was already very stable, we did not expect to improve $T_{\mathrm{m}}$, or decrease $f_{65}$ significantly, but rather we aimed to reduce the flexibility and conformational entropy of the native ensemble, thus also increasing its unfolding cooperativity. It was anticipated that this may then in some cases lead to slower aggregation, and therefore, identify potential hotspot surface loops whose flexibilities most strongly impact on aggregation.

The flexible regions of the A33 Fab were first identified by combining molecular dynamics simulation of A33 Fab, and B-factor analyses of multiple protein structures of high sequence similarity. Residues in the most flexible regions were mutated towards those where Rosetta predicted a lower $\Delta \Delta G_{\mathrm{ND}}$. One additional variant was constructed that was predicted by Rosetta to be the most stabilising, although it was in a region of low flexibility. A set of variants, predicted by Rosetta to be destabilising, were also prepared as negative controls to validate the design strategy. Of all designs, twelve stable variants and five unstable ones could be expressed sufficiently for study along with the wild type. The conformational stabilities and aggregation rates of the eighteen Fab variants were compared at a condition $(\mathrm{pH} \mathrm{4,} 200 \mathrm{mM}$ ionic strength, $65^{\circ} \mathrm{C}$ ), where $f_{65}<0.05$ for wild-type, and that was therefore, close to the limits of any correlation between aggregation rate and $T_{\mathrm{m}}$ or $f_{65}{ }^{36}$. Therefore, even if mutations had increased $T_{\mathrm{m}}$ and decreased $f_{65}$, they would not be expected to decrease the rate of aggregation significantly through a global unfolding mechanism. Instead, the 
mutational scanning led to increased cooperativity of unfolding associated with decreased conformational flexibility, and also determined that the aggregation rate was sensitive to the local flexibility of the heavy chain C-terminus in particular.

\section{MATERIALS AND METHODS}

\section{Analysis of Residue Flexibility}

Molecular Dynamics Simulation

The homology model of wild type A33 Fab was built from the crystal structure of human germline antibody 5-51/O12 (PDB ID 4KMT) and the amino-acid sequence of A33 Fab (See SI). The molecular dynamics simulation was conducted in Gromacs ${ }^{14}$. The protonation states of chargeable residues at $\mathrm{pH} 4$ were determined by uploading the PDB file to PDB2PQR web service ${ }^{39,40}$. The Fab PDB file was initially converted to a topology file with its five inter/intra-disulfide bonds retained. An OPLS-AA/L allatom force field was selected and protonation status was manually adjusted. The Fab was centred into a cubic box at least $1 \mathrm{~nm}$ away from the edge of the box. The box was filled with water molecules as solvent. The entire solution box was neutralised and adjusted to an ionic strength of $200 \mathrm{mM}$ by adding $\mathrm{Na}^{+}$and $\mathrm{Cl}^{-}$. The structure was then subjected to an energy minimisation step, and equilibrated at $300 \mathrm{~K}$ and atmospheric pressure for more than 40 ns. Jobs were submitted to UCL Legion High-Performance Computing Facility (Legion@UCL). At least three repeats were conducted to validate if data was reproducible. The root-mean square fluctuation (RMSF) data was exported 
based on trajectories beginning from 20 ns to allow for relaxation at the beginning of the simulation.

\section{B-factor}

Multiple homologous structures with 53-90\% sequence identities to A33 Fab, were retrieved from Protein Data Bank ${ }^{41}$, and aligned to A33 Fab in order to infer the flexibility across all residues. All the PDB files were modified so that only one set of light chain and heavy chain remained. Due to the crystallisation uncertainties for excessive thermal motion of certain residues, some residues were not displayed in their PDB files but shown in their sequence files. As a result, only residues present in PDB files could be used for sequence alignment with A33 Fab. Therefore, instead of using the FASTA sequence file, the actual sequence information was extracted from the PDB for heavy chain and light chain separately, and the sequence outputs from all human Fab PDBs were aligned with that of A33 Fab using BioEdit ${ }^{42}$.

For each sequence, the B-factors from each atom in the same residue were averaged and assigned to that residue. The B-factors within each protein were normalised into a distribution with average 0 and standard deviation $1^{21}$. Residue-averaged B-factors for each protein were aligned according to the sequence alignment. Only the B-factors for which there was a residue within the A33 Fab sequence were retained. The average B-factors for each A33 Fab residue were calculated by averaging the B-factors obtained at the same positions from each homologous structure. To reduce the noise, the Bfactors were window-averaged across 5 residues.

\section{In silico Mutagenesis and $\Delta \Delta G$ Calculation by Rosetta}


Point mutations were modelled into the homology model using Rosetta method "ddg_monomer" ${ }^{\prime 3}$ (see SI). Each of the 442 residues in the PDB structure was mutated into the other 19 amino-acid residues. In total, 8398 structures with single mutations were created. The "ddg_monomer" jobs were submitted to UCL Legion HighPerformance Computing Facility (Legion@UCL) with Rosetta Version 2015.31.58019. After mutating, the change in stability $(\Delta \Delta G)$ induced by each point mutation, was calculated with reference to the original wild type model.

\section{Design of Stable and Unstable Variant Candidates}

After the identification of flexible regions and the in-silico mutagenesis, variant candidates were selected for expression in the wet lab. Variants were categorised into stable and unstable classes.

\section{Stable Variant Candidates}

For stable variants, they were designed based on a combined analysis of B-factor, RMSF and $\Delta \Delta G$. The hinge regions in the heavy chains had no available B-factor values, but their RMSF values were very high. It was suggested that the hinge regions accounted for the flexibility and instability of an $\operatorname{IgG}$, and switching it to another subclass could potentially improve the formulation stability while maintaining its binding affinity ${ }^{5}$. Thus, the last two hinge residues, HC-A227 and HC-A228, were selected for mutation. For the other regions (residues 1 to 436), both B-factor and RMSF were considered. Because the scales of B-factor and RMSF were not comparable, the B-factor and RMSF values were firstly normalised to between 0 and 1 according to Equation 1. Individual residues were then ranked based on the product of 
normalised B-factor and RMSF, and the top 5 residues were selected. For a total of 7 selected residues, each was mutated into the other three amino acids predicted by Rosetta to have the lowest $\Delta \Delta G$ values from across all 19 candidates. Three mutations were selected because Rosetta is expected to fit with $69 \%$ prediction accuracy ${ }^{43}$, so theoretically, three mutations should yield at least one stable variant. An additional 5 mutations were selected based only on the lowest $\Delta \Delta G$ values predicted by Rosetta, regardless of the flexibility of the target site.

Normalised value

$$
=\frac{\text { Raw value }- \text { Min }}{\text { Max }- \text { Min }}
$$

Equation 1

In order to design the variants only based on B-factor, RMSF and $\Delta \Delta G$ values without losing or introducing new features, several additional filter criteria were applied. Mutations were avoided that would introduce a cysteine, remove a disulphide bond, or disrupt salt bridges. In addition, mutations were avoided in the Complementarity Determining Regions (CDR) of the Fab as this would affect Fab function, and guide mutations to regions that could be potentially useful in any Fab generated by industry. Unstable variant candidates

The unstable variants were designed from only those candidates with the highest $\Delta \Delta G$ values as predicted by Rosetta. As above, the designed variants avoided mutations from and to cysteine, salt bridge modifications, and the CDR regions of Fab. 
As the top 25 variants with highest $\Delta \Delta G$ were mutations into proline, 14 of which were glycine to proline mutations, variants ranked after the first 25 were also selected to expand the range of mutation types.

\section{In vitro Site-Directed Mutagenesis and Transformation}

Site-directed mutagenesis was conducted using QuikChange II XL Site-Directed Mutagenesis Kit (Agilent Technologies, US), and mutated plasmids transformed into One Shot TOP10 Chemically Competent E. coli (Thermo Fisher Scientific, UK), grown on $\mathrm{Tet}^{+} \mathrm{LB}$ agar. Single colonies were isolated for plasmid purification and sequencing. Correct plasmids were retransformed into W3110 E. coli, and glycerol stocks prepared for subsequent fermentation.

\section{Fab expression, purification and buffer exchange}

The fermentation protocol described previously ${ }^{36}$ was scaled-down to match the 180 ml DASbox Mini Bioreactor (Hamburg, Germany). The same purification protocol was retained, with protein aliquots snap-frozen and stored at $-80{ }^{\circ} \mathrm{C}$. Fab formulations were prepared and equilibrated at $4{ }^{\circ} \mathrm{C}$ one day prior to aggregation kinetics and thermal stability analyses. The frozen sample of Fab was thawed from the $-80{ }^{\circ} \mathrm{C}$ freezer and filtered through Anotop $250.02 \mu \mathrm{m}$ syringe filters (GE Healthcare, Buckinghamshire, UK) to remove aggregates, then buffer exchanged to MilliQ water, and concentrated to $2 \mathrm{mg} / \mathrm{mL}$ with $30 \mathrm{kDa}$ cut-off Vivaspins (Generon Ltd, Bershire, UK), and stored at $4{ }^{\circ} \mathrm{C}$.

Thermal Stability Analysis 
The thermal stability analysis was conducted using the UNit (Unchained Labs, UK). Fab samples were formulated in triplicates at $1 \mathrm{mg} / \mathrm{ml}, \mathrm{pH} 4$ of $20 \mathrm{mM}$ sodium citrate with $\mathrm{NaCl}$ to bring the total ionic strength to $200 \mathrm{mM}$. To achieve this, $20 \mu \mathrm{L}$ of 2 $\mathrm{mg} / \mathrm{mL}$ Fab in water was added to $20 \mu \mathrm{L}$ double-concentrated stock buffer for each variant. $9 \mu \mathrm{L}$ was pipetted into the cuvette for each sample and loaded into the UNit. Step-wise heating was applied from $20-90^{\circ} \mathrm{C}$ at $1{ }^{\circ} \mathrm{C} /$ step. The van't Hoff thermal parameters $\Delta H_{\mathrm{vh}}, \Delta S_{\mathrm{vh}}$ and thermal unfolding temperature $\left(T_{\mathrm{m}}\right)$ at which $50 \%$ of protein population was unfolded, were determined from the barycentric mean (BCM) of protein intrinsic fluorescence spectra at $280-460 \mathrm{~nm}$, at each temperature, by fitting to the van't Hoff equation (Equation 2 and Equation 3) ${ }^{44,45}$. The fraction of unfolded protein $\left(f_{\mathrm{T}}\right)$ at any temperature $\mathrm{T}$ was calculated from Equation $4 . T_{\text {on }}$ was defined as the temperature $\mathrm{T}$ at which $2 \%$ protein was unfolded (i.e. $f_{\mathrm{T}}=0.02$ ) using Equation 5 derived from both Equation 2 and Equation 4.

$$
\begin{array}{ll}
I_{T}=\frac{\left(I_{N}+a T\right)+\left(I_{D}+b T\right) \exp \left[\frac{\Delta H_{v h}}{R}\left(\frac{1}{T_{m}}-\frac{1}{T}\right)\right]}{1+\exp \left[\frac{\Delta H_{v h}}{R}\left(\frac{1}{T_{m}}-\frac{1}{T}\right)\right]} & \text { Equation 2 } \\
\Delta S_{v h}=\frac{\Delta H_{v h}}{T_{m}} & \text { Equation 3 } \\
f_{T}=\frac{I_{T}-I_{N}-a T}{I_{D}+b T-I_{N}-a T}=\frac{\exp \left[\frac{\Delta H_{v h}}{R}\left(\frac{1}{T_{m}}-\frac{1}{T}\right)\right]}{1+\exp \left[\frac{\Delta H_{v h}}{R}\left(\frac{1}{T_{m}}-\frac{1}{T}\right)\right]} & \text { Equation 4 }
\end{array}
$$




$$
T_{\text {on }}=\frac{\Delta H_{v h}}{\Delta S_{v h}-R \ln \left(\frac{f_{T}}{1-f_{T}}\right)} \quad\left(\operatorname{set} f_{T}=0.02\right)
$$

Equation 5

\section{Aggregation Kinetics}

Double-concentrated stock buffer was prepared as above for the thermal stability analysis, and mixed with $2 \mathrm{mg} / \mathrm{ml} \mathrm{Fab}$ in MilliQ water, to achieve a final concentration of $1 \mathrm{mg} / \mathrm{ml} \mathrm{Fab}$ in $20 \mathrm{mM}$ sodium citrate, with $\mathrm{NaCl}$ to bring the total ionic strength to $200 \mathrm{mM}$. Each sample was aliquoted into $20 \mu \mathrm{L}$ in a $0.2 \mathrm{~mL}$ thin-walled RNase-free PCR tube (Fisher Scientific, UK), and placed onto a thermal cycler (Bio-Rad C1000 Touch, UK) set at $65^{\circ} \mathrm{C}$. Samples were sacrificed in triplicates for each variant every 1 or $5 \mathrm{~min}$, quenched on ice for $5 \mathrm{~min}$, and centrifuged at $15,000 \mathrm{rpm}, 4{ }^{\circ} \mathrm{C}$ for $15 \mathrm{~min}$, from which $15 \mu \mathrm{L}$ supernatant was transferred into a HPLC vial insert, and $5 \mu \mathrm{L}$ injected into an Agilent Zorbax Bio Series GF-250 SEC-HPLC column (Agilent, Berkshire, UK) to quantify the monomer. The SEC-HPLC was operated at a flowrate of $1 \mathrm{ml} / \mathrm{min} 200 \mathrm{mM}$ sodium phosphate, $\mathrm{pH} 7$ on an Agilent 1200 HPLC system (Cheshire, UK), for $4.5 \mathrm{~min}$ in each cycle. Calibration curves were established prior to each batch of analyses.

An exponential function was used to fit the kinetics of monomer loss using Equation 6 , in which $A$ and $k$ are the coefficients, y is the monomer retention normalised from 0 to 1 , and $t$ is the incubation time. The first derivative of Equation 6 is shown in Equation 7 , in which the absolute initial aggregation rate is " $A * k$ " when $t=0$. 


$$
\begin{array}{ll}
\mathrm{y}=A \times e^{(-k t)} & \text { Equation 6 } \\
\frac{d(y)}{d(t)}=-A \times k \times e^{(-k t)} & \text { Equation 7 }
\end{array}
$$

\section{RESULTS AND DISCUSSION}

\section{Identifying Flexible Regions}

The mutational impacts upon protein stability have been extensively reviewed ${ }^{11,46-48}$. Proteins can become conformationally stabilised, such as via improved hydrophobic interactions and packing density in the protein interior, or they can be colloidally stabilised, due to increased surface net charge or decreased hydrophobicity. The present work aimed to reduce the local dynamics that contribute to conformational instability and also potentially to aggregation kinetics, and so the most flexible regions were identified first. The RMSF data by residue, from molecular dynamics simulation of the wild type Fab are shown in Figure 1(A). It can be seen from the standard error of the mean at each residue, that the deviation between simulation repeats was greatest when the RMSF also had the highest values. RMSF was broadly dependent on the type of secondary structure, where loop regions often had high RMSF $(0.137 \pm 0.052 \mathrm{~nm}) ; \beta$ strand regions had relatively low RMSF values $(0.093 \pm 0.023 \mathrm{~nm})$; and helical regions also had high RMSF on average $(0.164 \pm 0.096 \mathrm{~nm})$.

The PDB IDs of accessed homologous human Fabs are shown in the Supplementary Information. The normalised B-factors for each residue position, after window averaging, is shown in Figure 1(B), and had a similar sequence dependence as the 
RMSF. This implies that B-factors from the crystal structures of Fab, which reflect a disorder in vitro, can be simulated by molecular dynamics in silico. In order to verify that both independent measurements achieve similar flexibility results for the protein residues, RMSF and B-factors were plotted against each other and gave a modest correlation $\left(\mathrm{R}^{2}=0.56\right)$ in Figure $1(\mathrm{C})$.

B-factors also showed that the $\beta$-strand structures were the most rigid, whereas the few helical structures were relatively flexible. The loop regions covered almost the entire magnitude for both RMSF and B-factors, but also showed the highest flexibility in some locations. While proteins with more $\beta$-sheet regions are more prone to forming aggregates $^{49,50}$, the $\beta$-strands were relatively less flexible than helices and loops, largely as they were also less solvent exposed in the Fab structure. Therefore, the conformation and flexibility of surrounding structure is likely to play a key role in controlling the solvent exposure of the $\beta$-strands, and hence their ability to form aggregates ${ }^{51}$. It is interesting to see that only a small proportion of all loops exhibit significantly high flexibility, yet the five most flexible regions are loops. This result indicated that mutagenesis could be targeted to residues with high flexibility in loop regions as determined by both the RMSF and B-factor analyses, to decrease the local flexibility through increased interactions. While not all flexible loops would be expected to influence the aggregation rate, this represents a substantially reduced set of targets in the protein that could potentially be mutated to minimise aggregation. 

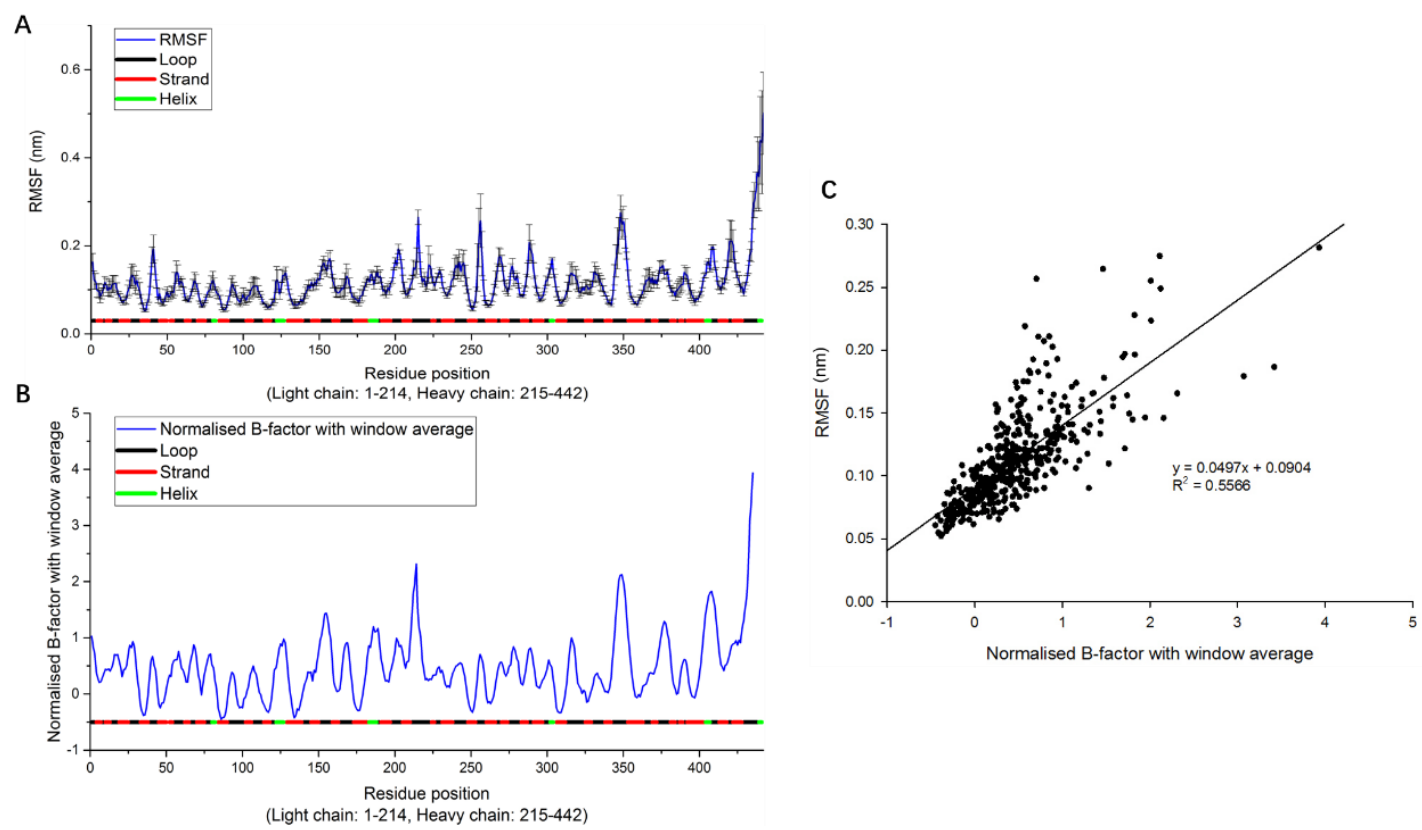

Figure 1. Residue-dependent flexibility of Fab. (A) Average RMSF for wild-type A33 Fab at $\mathrm{pH}$ 4, $200 \mathrm{mM}$ ionic strength. Error bars are the standard error of the mean from three repeat simulations. (B) Normalised B-factors averaged from 26 homologous structures, and window averaged over 5 contiguous residues. The colour bar denotes secondary structure type as determined by PyMol, with loop (black), strand (red) and helix (green) regions. (C) Correlation between RMSF (A) and B-factor (B).

\section{In silico Single Point Mutagenesis for $\Delta \Delta G_{\mathrm{ND}}$}

The homology model for the wild type is shown in the supplementary information. After in-silico mutagenesis, 8398 mutations were generated. Their $\Delta \Delta G_{\mathrm{ND}}$ values, in arbitrary Rosetta Energy Units (REU), and their frequency distribution are plotted in the supplementary information. It can be seen that most of the mutations had $\Delta \Delta G_{\mathrm{ND}}$ values of close to 0 , which implied that most single mutations were predicted to have little impact on global conformational stability. Moreover, negative $\Delta \Delta G_{\mathrm{ND}}$ values were 
all greater than -9.4 REU, whereas positive $\Delta \Delta G_{\mathrm{ND}}$ values extended much further with a maximum value of $235 \mathrm{REU}$. This result implied that the A33 wild type structure was already relatively stable within the accessible sequence space for single mutations, which was not surprising given that it was obtained after significant previous selection and engineering as a potential therapeutic ${ }^{52}$. With a $\Delta G_{\mathrm{ND}}$ of around -1100 REU, an improvement by up to $-9.4 \mathrm{REU}$, would be expected to provide less than $1 \%$ stabilisation of the global structure. Conversely, the destabilising variants were expected to exhibit greater detrimental effects, of up to $20 \%$ loss in $\Delta G_{\mathrm{ND}}$. These included polar or ionisable mutations in the protein core, hydrophobic mutations on the outer surface, steric hindrance caused by large amino acid substitutions, and the removal of salt bridges and hydrogen bonds.

There were 2386 potentially stabilising variants (i.e. $\Delta \Delta G_{\mathrm{ND}}$ values lower than 0 ). To investigate whether they were biased towards particular secondary structure types, the lowest $\Delta \Delta G$ values for each residue were plotted by colour on the Fab structure in Figure 2. The blue regions in the figure represent stabilising effects, and the red ones indicate where no improvement could be made by any of the 19 mutations. Most of the blue regions were located within $\beta$-sheet structures (e.g. LC-N137, LC-S176), while some were in the turn or random coil regions (e.g. HC-L61, HC-T135), though this appears to simply reflect the relative abundance of each secondary structure type. 


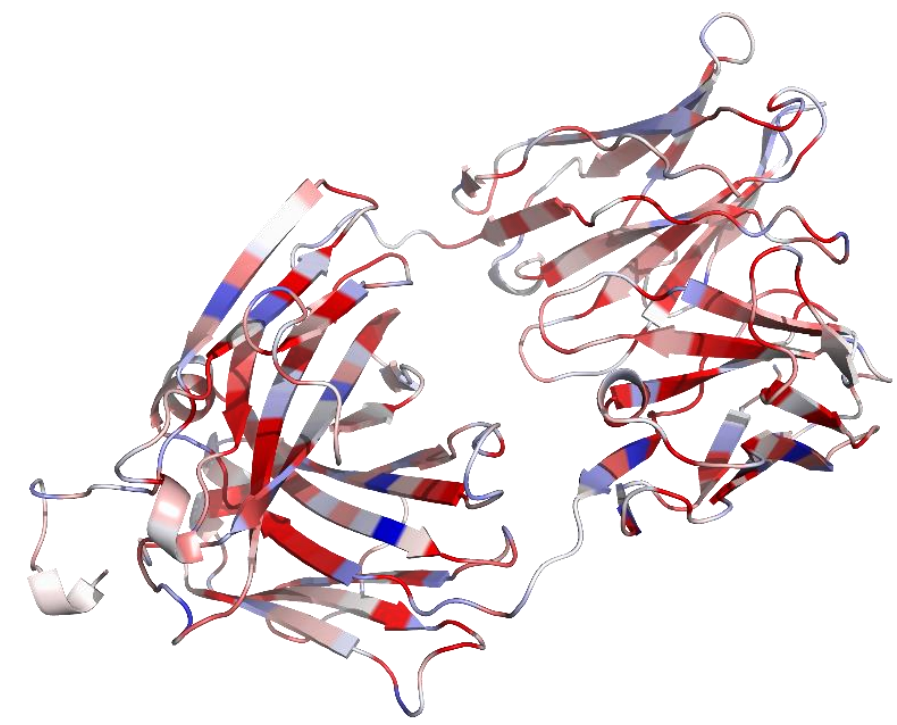

Figure 2 A blue-white-red plot to represent the locations of stable variants.

For each residual position, there are 19 mutations. The lowest $\Delta \Delta G_{\mathrm{ND}}$ value of the 19 mutations is plotted in gradient colours from blue to white to red, which demonstrates the $\Delta \Delta G_{\mathrm{ND}}$ from lowest negative value to 0 . Where the lowest $\Delta \Delta G_{\mathrm{ND}}$ was greater than 0 , a value of 0 was retained to indicate no improvement.

\section{Designed Variants and Laboratory Production}

Initially, 26 stable variants and 9 unstable variants were selected for generation and expression. Some proved difficult to generate by site direct mutagenesis, and others did not grow or express sufficiently well in cell culture to obtain sufficient material for analysis. A total of 12 stable variants and 5 unstable variants were studied. Rosetta predicted that the single variant variants finally studied would not stabilise the original molecule by more than $1 \%$ in terms of global unfolding $\left(\Delta \Delta G_{\mathrm{ND}}\right)$, whereas five variants 
could lead to significant destabilisation of between $3 \%$ and $21 \%$ of the $\Delta \Delta G_{\mathrm{ND}}$ of wild type (Table 1).

Table 1 Predicted $\Delta \Delta G_{\mathrm{ND}}$ of designed stable and unstable variants

\begin{tabular}{|c|c|c|c|c|c|}
\hline & Variant ID & $\Delta \Delta G_{\mathrm{ND}}$ & $\begin{array}{l}\Delta \Delta G_{\mathrm{ND}} / \Delta G_{\mathrm{WT}} \\
(\%)^{(1)}\end{array}$ & $\begin{array}{l}\text { RMSF } \\
(\mathrm{nm})^{(2)}\end{array}$ & B-factor ${ }^{(3)}$ \\
\hline & Wild type & 0.0 & $\mathrm{~N} / \mathrm{A}$ & $\mathrm{N} / \mathrm{A}$ & $\mathrm{N} / \mathrm{A}$ \\
\hline \multirow{12}{*}{ Stable } & HC-A227E & -4.4 & $0.39 \%$ & 0.44 & $\mathrm{~N} / \mathrm{A}$ \\
\hline & HC-A228H & -3.5 & $0.31 \%$ & 0.50 & $\mathrm{~N} / \mathrm{A}$ \\
\hline & HC-T135Y & -2.6 & $0.23 \%$ & 0.25 & 2.12 \\
\hline & HC-S134Y & -6.0 & $0.53 \%$ & 0.27 & 2.11 \\
\hline & HC-S134M & -5.3 & $0.46 \%$ & 0.27 & 2.11 \\
\hline & HC-S134P & -4.0 & $0.35 \%$ & 0.27 & 2.11 \\
\hline & HC-S136G & -4.3 & $0.38 \%$ & 0.26 & 2.01 \\
\hline & HC-S219Y & -5.0 & $0.44 \%$ & 0.18 & 3.07 \\
\hline & LC-L154A & -3.8 & $0.33 \%$ & 0.15 & 1.42 \\
\hline & LC-S176W ${ }^{(4)}$ & -8.8 & $0.78 \%$ & 0.07 & -0.29 \\
\hline & HC-G194H & -5.4 & $0.48 \%$ & 0.20 & 1.83 \\
\hline & HC-T197L & -4.0 & $0.35 \%$ & 0.14 & 1.31 \\
\hline \multirow{5}{*}{ Unstable } & LC-A153P & 235.7 & $-20.82 \%$ & 0.16 & 1.23 \\
\hline & LC-G66P & 163.3 & $-14.42 \%$ & 0.09 & 0.60 \\
\hline & LC-G200W & 40.8 & $-3.60 \%$ & 0.17 & 0.75 \\
\hline & HC-V215W & 39.1 & $-3.44 \%$ & 0.09 & 1.30 \\
\hline & HC-G178P & 86.0 & $-7.57 \%$ & 0.13 & 0.46 \\
\hline
\end{tabular}


(1) Rosetta "ddg_monomer" simulated 50 conformations for wild type and each variant, calculated $\Delta G_{\mathrm{ND}}$ for each conformation, and then determined $\Delta \Delta G_{\mathrm{ND}}$ from their respective minimum values, as $\Delta G_{\mathrm{mut}}-\Delta G_{\mathrm{WT}}$. Positive values of $\Delta \Delta G_{\mathrm{ND}} / \Delta G_{\mathrm{WT}}$ are predicted to be stabilising, and negative values destabilising mutations. (2) The RMSF values of wild type at the mutational sites, also shown in Figure 1(A), with the average of all the residues at 0.12. (3) The window averaged and normalised B-factor from homology models, also shown in Figure 1(B), with the average of all the residues at 0.47. (4) LC-S176W was the only variant designed based on the highest stabilisation as predicted by Rosetta alone.

\section{Thermal Stability Measurement for $\boldsymbol{T}_{\mathrm{m}}, \boldsymbol{T}_{\mathrm{on}}$ and Fraction of Unfolded State $\boldsymbol{f}_{\mathrm{T} 65}$}

After the expression of designed variants, their conformational stability was assessed through different thermal stability measures. We selected a formulation condition of 1 $\mathrm{mg} / \mathrm{ml} \mathrm{Fab}$ in $20 \mathrm{mM}$ sodium citrate, $\mathrm{pH} \mathrm{4}$, at an ionic strength of $200 \mathrm{mM}$, as this partially unfolded the wild-type protein at $65^{\circ} \mathrm{C}$ by $6 \%{ }^{36}$, and to led to aggregation on a practical timescale. These conditions were used for both thermal stability measurement and aggregation kinetics.

\section{Determination of $T_{\mathrm{m}}$}

To determine experimentally the stabilising or destabilising effects of each mutation, the thermal transition mid-point temperatures $\left(T_{\mathrm{m}}\right)$ of variants were measured by intrinsic protein fluorescence. As shown in Figure 3(A), all the stabilising variants ranged in a narrow window with $T_{\mathrm{m}}$-values from $70.2-72.5^{\circ} \mathrm{C}$. This was consistent with the Rosetta predictions, and confirmed that the A33 Fab structure was already relatively stable to global unfolding, as a result of significant selection and engineering as a potential therapeutic. All five of the destabilising variants had significantly lower $T_{\mathrm{m}}$-values than the wild type, ranging from $67.1^{\circ} \mathrm{C}$ for LC-A153P to $60.2{ }^{\circ} \mathrm{C}$ for the least stable variant LC-G66P. 


\section{Determination of $f_{\mathrm{T} 65}$}

The coefficients derived from $T_{\mathrm{m}}$ curve-fitting (Equation 2) were used to calculate the fraction of unfolded protein $f_{\mathrm{T}}$ at a particular temperature $\mathrm{T}$ (Equation 4), which has a sigmoidal dependence on temperature. When the temperature is much lower or much higher than the $T_{\mathrm{m}}$, the $f_{\mathrm{T}}$ approaches to 0 or 1 , respectively. The dependence of $f_{\mathrm{T}}$ on both $T_{\mathrm{m}}$ and $\Delta H_{\mathrm{vh}}$, means that a good correlation between $T_{\mathrm{m}}$ and $f_{\mathrm{T}}$ exists only at temperatures close to $T_{\mathrm{m}}$. In this work, the incubation temperature was deliberately chosen at $65^{\circ} \mathrm{C}$, which is at most $7{ }^{\circ} \mathrm{C}$ from the $T_{\mathrm{m}}$ of all variants, and thus a good correlation between $T_{\mathrm{m}}$ and $f_{\mathrm{T} 65}$ is expected.

As shown in Figure 3(A), wild-type was $4.7 \%$ unfolded at $65^{\circ} \mathrm{C}$, and most of the variants predicted to be stabilising were similarly $3.5-5.9 \%$ unfolded, except HCT197L (10.6\%). Three variants predicted to be unstable, LC-A153P, LC-G200W and HC-V215W were 25-35\% unfolded, while HC-G178P unfolded 62\% and LC-G66P unfolded $86 \%$. Overall this created a diverse range of variants unfolded from $3.5-86 \%$ at $65{ }^{\circ} \mathrm{C}$, enabling the role of global unfolding on aggregation kinetics at $65^{\circ} \mathrm{C}$ to be deconvoluted from other effects below.

\section{Determination of $T_{\mathrm{on}}$}

Another useful parameter is the unfolding onset temperature $T_{\text {on }}$ (Equation 5), at which $2 \%$ of the protein is unfolded ( $\operatorname{set} f_{\mathrm{T}}=0.02$ ). Previous analyses of aggregation kinetics for $\mathrm{A} 33 \mathrm{Fab}^{36}$, and $\mathrm{GCSF}^{53,54}$, indicated that the mechanism of aggregation at $f_{\mathrm{T}}<0.01$ was predominantly from relatively rare native-like states, and not due to global unfolding. This was consistent with previous suggestions that protein monomers would 
form a nucleus before associating into larger oligomers ${ }^{55}$, and that this nucleus is very likely to be generated through the effective collision between the exposed hydrophobic residues from partially unfolded proteins. Hence, $T_{\text {on }}$ (at which $\left.f_{\mathrm{T}}=0.02\right)$, represents the temperature above which global unfolding may begin to play a more significant role in aggregation. The difference between $T_{\mathrm{on}}$ and $T_{\mathrm{m}}$, is an indirect measure of the cooperativity of unfolding, which is more directly reported by $\Delta S_{\mathrm{vh}}$ in Equation 3. This is potentially influenced by the degree of local dynamics in the native structure, and hence the breadth of the native-state ensemble, from which global unfolding occurs. Therefore, if dynamics in the native-state ensemble have a role in aggregation, then $T_{\text {on- }}$ values or $\Delta S_{\mathrm{vh}}$ could potentially provide a valuable indication of the propensity to aggregate at temperatures well below $T_{\mathrm{m}}$.

Figure 3(A) shows the variant $T_{\text {on-values, and the significance of their differences to }}$ that of the wild type. The variants predicted to be stable had similar $T_{\mathrm{on}}$, and mostly ranged from $62.7-63.8{ }^{\circ} \mathrm{C}$ (except HC-T197L), with HC-T135Y significantly higher than the wild type. Variant LC-S176W had an unusual thermal unfolding behaviour, which is detailed in the SI. Not surprisingly, all of the unstable variants, had significant drops of $3.5-12.7^{\circ} \mathrm{C}$ in $T_{\text {on }}$ compared to wild type. In general, variants predicted to be most stable by Rosetta, had higher $T_{\mathrm{m}}$ and $T_{\mathrm{on}}$, and lower $f_{\mathrm{T} 65}$ compared to those predicted to be unstable. 


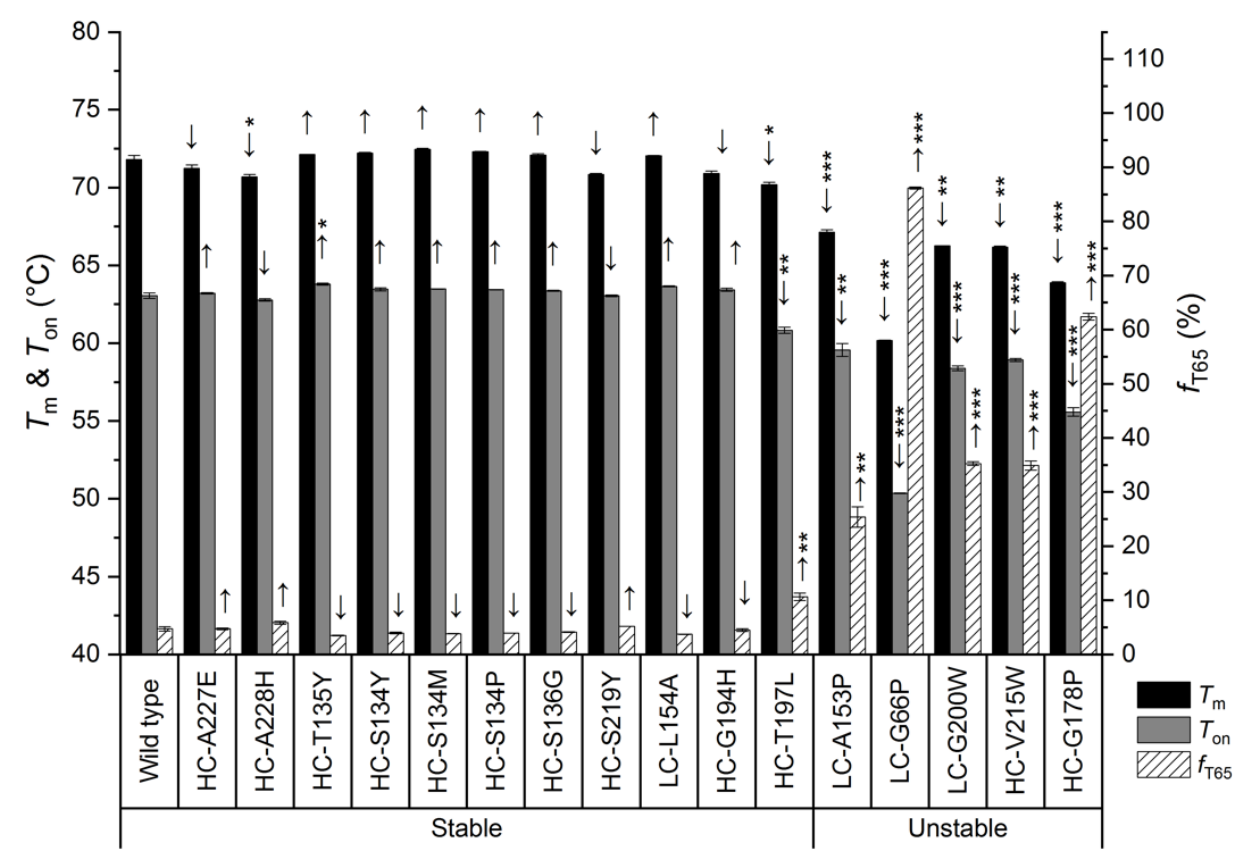

A

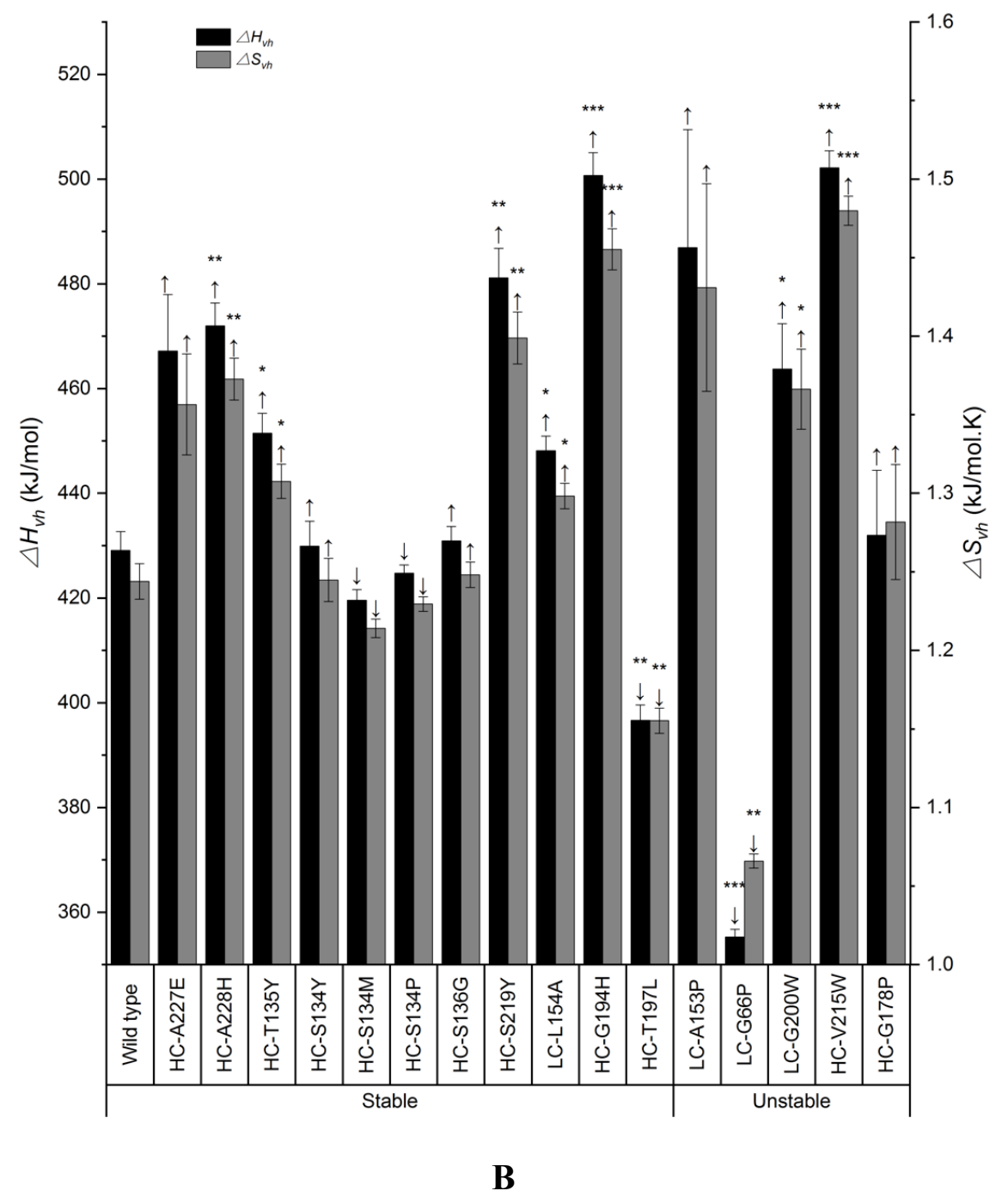


Figure 3 (A) The melting temperature ( $T_{\mathrm{m}}$, dark bars), temperature when $2 \%$ was unfolded ( $T_{\mathrm{on}}$, grey bars) and fraction of unfolding at $65^{\circ} \mathrm{C}\left(f_{\mathrm{T} 65}\right.$, tilt-line bars $)$ as measured by UNit in triplicates at $1 \mathrm{mg} / \mathrm{ml}, \mathrm{pH} 4$ of $20 \mathrm{mM}$ sodium citrate with $\mathrm{NaCl}$ to bring the total ionic strength to $200 \mathrm{mM}$. (B) The enthalpy and entropy changes at the midpoint of transition, $\Delta H_{\mathrm{vh}}$ and $\Delta S_{\mathrm{vh}}$, derived from van't Hoff analysis. The unusal melting curve for LC-S176W precluded the determination of enthalpy and entropy for this variant. All error bars were standard error of the mean (SEM). Arrow $\uparrow$ or $\downarrow$ indicated an increase or decrease in average values of variants compared to the wild type. Two-sample t-test assuming unequal variances were performed between A33 wild type and other variants $(* * * \mathrm{p}<0.001, * * \mathrm{p}<0.01, * \mathrm{p}<0.05)$.

Van't Hoff analysis for the enthalpy and entropy change

The changes in van't Hoff enthalpy and entropy ( $\Delta H_{\mathrm{vh}}$ and $\left.\Delta S_{\mathrm{vh}}\right)$ between native and unfolded states, were determined for the variants at the $T_{\mathrm{m}}$ using the van't Hoff analysis $^{44,45}$, as shown in Figure 3(B). As the $T_{\mathrm{m}}$ values did not vary significantly between variants, $\Delta H_{\mathrm{vh}}$ and $\Delta S_{\mathrm{vh}}$. were highly correlated. Four of the stable variants had no significant change in $\Delta H_{\mathrm{vh}}$ and $\Delta S_{\mathrm{vh}}$, which implied they had comparable stability compared to the wild type. Six variants, namely HC-A227E, HC-A228H, HC-T135Y, HC-S219Y, LC-L154A and HC-G194H, saw significant increases in $\Delta H_{\mathrm{vh}}$ and $\Delta S_{\mathrm{vh}}$, which indicated decreased conformational flexibility via lower $S_{N}$ values for the native ensemble than the wild type, assuming that both wild type and the variants 
retained a similar $S_{U}$ for the unfolded ensemble. This demonstrates that targeted stabilisation to rigidify flexible regions, can reduce the number of conformational states populated in the native ensemble, as measured by an increase in unfolding cooperativity $^{56}$. Only HC-T197L, of the designs intended to be stabilizing, saw a considerable reduction in $\Delta H_{\mathrm{vh}}$ and $\Delta S_{\mathrm{vh}}$, suggesting that this mutation increased the conformational entropy of the native state, concomitant with the observed decrease in $T_{\mathrm{m}}$.

For the variants designed to be unstable, four out of five had an increase in $\Delta H_{\mathrm{vh}}$ and $\Delta S_{\mathrm{vh}}$ while LC-G66P had a respective $17.2 \%$ and $13.7 \%$ decrease in $\Delta H_{\mathrm{vh}}$ and $\Delta S_{\mathrm{vh}}$, compared to the wild type. Nevertheless, their adversely decreased $T_{\mathrm{m}}$ would still be expected to dominate their behavior with respect to aggregation.

\section{Variants Stabilised Through Increased Cooperativity}

Compared to the wild type, no significant improvements in $T_{\mathrm{m}}$ were found for the variants predicted to be stable $(\mathrm{p}=0.45)$. However, HC-T135Y $(\mathrm{p}=0.04)$ and LC-L154A ( $\mathrm{p}=0.07$ ) had marked increases in their $T_{\text {on. }}$ As the designed mutations did not greatly enhance the thermal stability globally through $T_{\mathrm{m}}$, increases in $T_{\mathrm{on}}$ that decrease $T_{\mathrm{m}}-T_{\mathrm{on}}$ would imply improvements in unfolding cooperativity. Indeed, seven out of the twelve predicted stable variants had values of $T_{\mathrm{m}}-T_{\mathrm{on}}$ of $7.5-8.7^{\circ} \mathrm{C}$, compared to $8.8^{\circ} \mathrm{C}$ for wild type. These had correspondingly higher $\Delta S_{\mathrm{vh}}$ (Table 2), obtained from the $T_{\mathrm{m}}$ curve fitting (Equation 2 and Equation 3). Specifically, HC-A227E, HC-A228H, HCT135Y, HC-S219Y, LC-L154A and HC-G194H all had significant increase in $\Delta S_{\mathrm{vh}}$, confirming a decreased $S_{\mathrm{N}}$, and their increased unfolding cooperativity ${ }^{56}$. 
The A33 Fab structure is already very stable globally with a $T_{\mathrm{m}}$ of $71.8^{\circ} \mathrm{C}$, and lost only $1 \%$ of monomer to aggregation when stored at $4{ }^{\circ} \mathrm{C}$ for one year ${ }^{36}$. This may be the reason why for this particular protein, targeting single stabilising mutations to rigidify the most flexible loops, was more likely to increase the unfolding cooperativity, than to improve global stability to unfolding. This further suggests that for A33 Fab at least, a key feature of the protein that modulates unfolding cooperativity, outside of the main globally stabilising core interactions, is the flexibility of loops in the native state. Localised loop flexibility would manifest as an increased native-state ensemble, and hence a broadened range of unfolding trajectories ${ }^{57}$. Co-location of HC-A228H, HC-S219Y and HC-G194H in the hinge region of the heavy chain, implied that this region might be more involved in modulating the unfolding cooperativity than the flexible loop 134-136 of heavy chain, for which mutations did not significantly alter $\Delta S_{\mathrm{vh}}$

\section{Kinetics of Monomer Loss Under Conditions of Partial Global Unfolding}

The Fab variants were examined for their aggregation propensity at $65^{\circ} \mathrm{C}$, and in the same buffer as for the $T_{\mathrm{m}}$ measurements (20 mM sodium citrate, $\mathrm{pH} \mathrm{4,} 200 \mathrm{mM}$ ionic strength). According to Figure 3(A), this condition created a wide range in the fraction of unfolded states between the stable and unstable variants, spanning $3.5 \%$ to $86 \%$. Thus, it could be used to deconvolute the relative contribution of global protein 
unfolding, from other factors that also influence the aggregation rate, such as local structural flexibility.

We initially determined the rate-order for monomer loss from the dependence of the rate of monomer loss on protein concentration ( 1 to $8 \mathrm{mg} / \mathrm{ml}$ ) for the wild-type and four other Fab variants (Figure 4). All monomer loss curves fit well to single-exponential kinetics, and as shown in Figure 4(A), the initial rate-order for monomer loss was 0.59 to 0.74 , and rose sharply to 1 (Figure $4 \mathrm{C}$ ) as the reactions progressed. This indicated that monomer loss under the conditions and concentration range studied, was primarily rate-limited by a monomolecular reaction, such as partial protein unfolding. Deviation to a rate order less than 1 could result from several phenomena such as a second competing reaction, that was significant only at lower Fab concentrations and at earlier timepoints. This could result from a finite loss of monomer through early absorption to the vial surface, and would become less significant over time, or would consume only a relatively small proportion by mass at the higher concentrations ${ }^{58}$. Other effects like reversible dimerisation, macromolecular crowding, or viscosity might also result in lower than expected aggregation rates at higher concentrations, and cannot be ruled out. These effects would mean that the aggregation kinetics remain monomolecular (as observed from best fits to single exponentials), but also lead to the observed inverse dependence on protein concentration. 
A

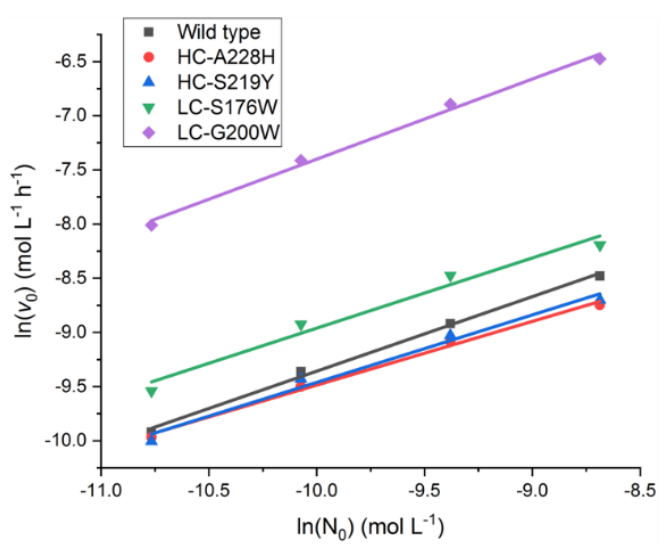

B

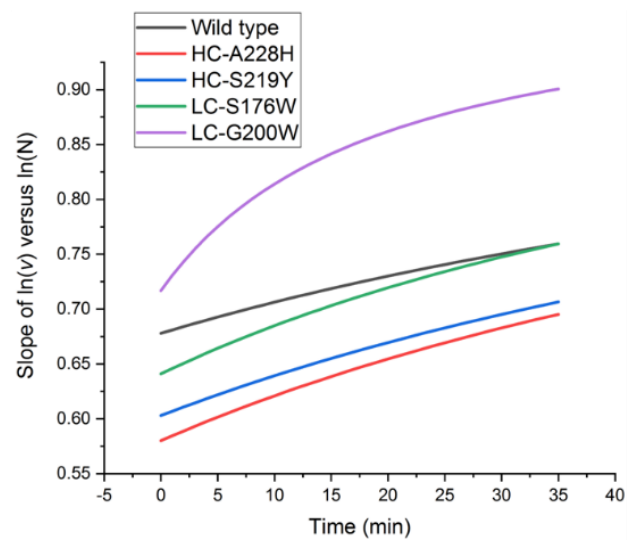

\begin{tabular}{|c|c|}
\hline Wild type & $\begin{array}{l}\ln \left(v_{0}\right)=0.6879 * \ln \left(N_{0}\right)-2.4783 \\
v=e^{-2.48} * N_{0}{ }^{0.69}\left(R^{2}=0.996\right)\end{array}$ \\
\hline $\mathrm{HC}-\mathrm{A} 228 \mathrm{H}$ & $\begin{array}{l}\ln \left(v_{0}\right)=0.5880 * \ln \left(N_{0}\right)-3.6060 \\
v=e^{-3.61} * N_{0}^{0.59}\left(R^{2}=0.995\right)\end{array}$ \\
\hline HC-S219Y & $\begin{array}{l}\ln \left(v_{0}\right)=0.6225 * \ln \left(N_{0}\right)-3.2355 \\
v=e^{-3.24} * N_{0}{ }^{0.62}\left(R^{2}=0.984\right)\end{array}$ \\
\hline LC-S176W & $\begin{array}{l}\ln \left(v_{0}\right)=0.6478 * \ln \left(N_{0}\right)-2.4829 \\
v=e^{-2.48} * N_{0}{ }^{0.65}\left(R^{2}=0.973\right)\end{array}$ \\
\hline LC-G200W & $\begin{array}{l}\ln \left(v_{0}\right)=0.7397 * \ln \left(N_{0}\right)-0.0037 \\
v=e^{-0.0037} * N_{0}{ }^{0.74}\left(R^{2}=0.994\right)\end{array}$ \\
\hline
\end{tabular}

C

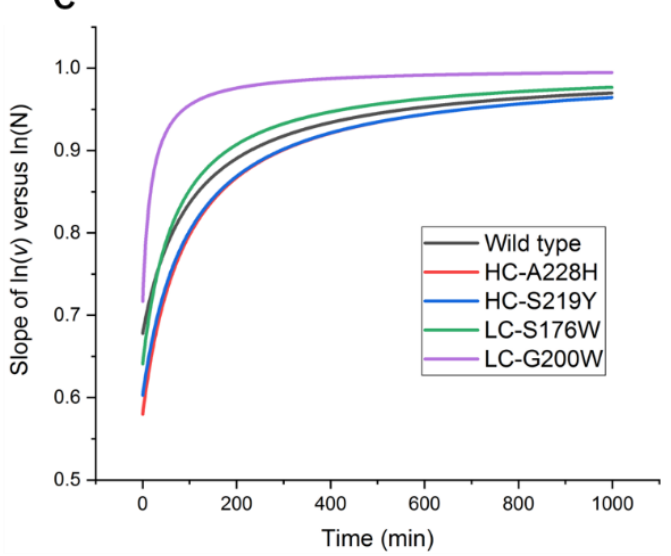

Figure 4 The correlation between the rate of monomer loss and protein concentration

(A) Wild type and four Fab variants were subjected to the same thermal treatment as the study for aggregation kinetics, but with initial concentration at 1, 2, 4 and $8 \mathrm{mg} / \mathrm{ml}$. $\mathrm{N}_{0}$ indicates the initial Fab concentration. The monomer retention curve was shown in SI. The initial rate of monomer loss was linearly correlated with the initial Fab concentration, both at logarithm scale.

(B \& C) Time-dependence of slope for $\ln \left(v_{t}\right)$ vs $\ln \left(\mathrm{N}_{t}\right)$, where $\ln \left(v_{t}\right)$ and $\ln \left(\mathrm{N}_{\mathrm{t}}\right)$ were calculated from Equation 6 and Equation 7, and the parameters A and $k$, obtained from 
the single-exponential fitting, over $35 \mathrm{~min}(\mathrm{~B})$ and $1000 \mathrm{~min}(\mathrm{C})$, for each of the four Fab concentrations.

Single exponential equations were used to fit the kinetics for loss of monomer of all variants as this provided the best fits. The aggregation kinetic constant $k$ from each exponential curve fit, and also the initial aggregation rates, are shown in Figure 5, to provide a quantitative comparison. A clear difference in the rate of monomer loss was observed between the stable and unstable variants. Wild type and most of the stabilising variants (except LC-S176W) lost 80\% of their monomers within 35 min, whereas the destabilising variants aggregated by the same extent in less than $10 \mathrm{~min}$. LC-G66P in particular lost all monomer within only $1.5 \mathrm{~min}$. The wild-type Fab aggregated with $k$ at $0.042 \mathrm{~min}^{-1}$, while most stable variants (except LC-S176W) had comparable kinetic constants, $k$. HC-S219Y and HC-G194H had $k 0.037 \mathrm{~min}^{-1}$, which was an improvement of over $11 \%$ compared to the wild type. All the unstable variants had $k$ more than $0.18 \mathrm{~min}^{-1}$, with LC-G66P fastest at $1.65 \mathrm{~min}^{-1}$, and 40 -fold higher than the rate for wild type.

The stabilising and destabilising effect of mutations in the variants was reflected similarly in their initial aggregation rate, expressed as $\ln (v)$ in $\%$ day $^{-1}$. Again, HCS219Y and HC-G194H reduced the initial rate by more than $12 \%$, while $\mathrm{HC}-\mathrm{A} 228 \mathrm{H}$ was slower by $6.5 \%$ compared to the wild type. None of the other stable variants had 
any noticeable improvement, while the unstable ones all had initial rates of monomer loss that were more than $340 \%$ that of wild type.

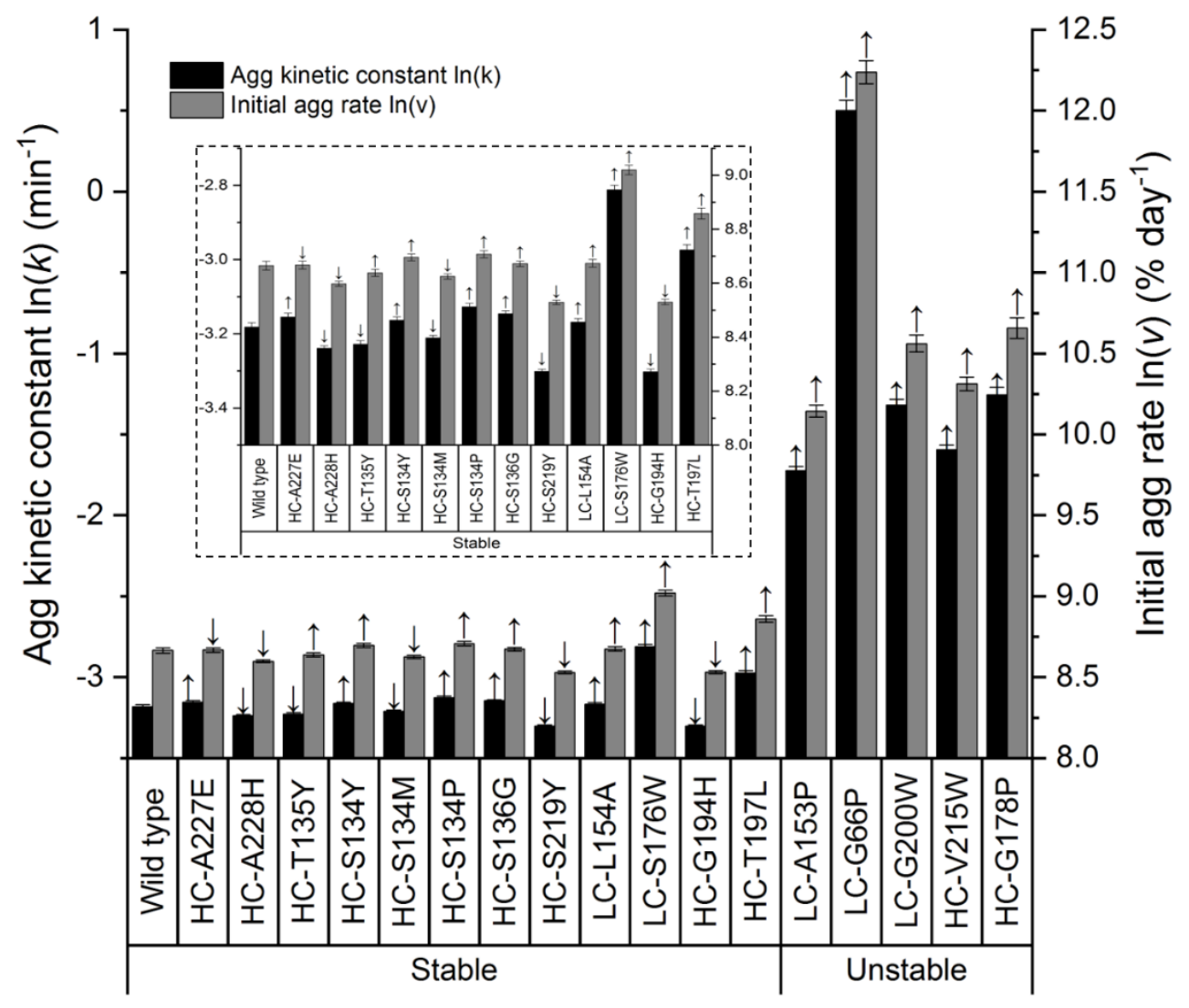

Figure 5 The aggregation kinetic constants and initial aggregation rates of A33 Fab wild type and designed variants.

The aggregation kinetic constant $\ln (k)$ and initial aggregation rates of A33 Fab wild type and designed variants. They were derived from the exponential equations, fitted from the monomer retention curves. Arrows $\uparrow$ or $\downarrow$ indicate increased or decreased values compared to those of wild type. Error bars were standard errors, calculated from curve-fitting derived parameters. Inset: Expanded view of stable variants only. 
Table 2 The thermal stability data and aggregation kinetic parameters for the variants

\begin{tabular}{|c|c|c|c|c|c|c|c|c|c|c|c|c|}
\hline & \multirow[b]{3}{*}{ Mutant ID } & \multirow{3}{*}{$\begin{array}{c}T_{\mathrm{m}} \\
\left({ }^{\circ} \mathrm{C}\right)\end{array}$} & \multirow{3}{*}{$\begin{array}{l}T_{\text {on }} \\
\left({ }^{\circ} \mathrm{C}\right)\end{array}$} & \multirow{3}{*}{$\begin{array}{l}f_{\mathrm{T} 65} \\
(\%)\end{array}$} & \multirow{3}{*}{$\begin{array}{c}\Delta H_{\mathrm{vh}} \\
\left(\mathrm{kJ} \mathrm{mol} \mathrm{mol}^{-1}\right)\end{array}$} & \multirow{3}{*}{$\begin{array}{c}\Delta S_{\mathrm{vh}} \\
\left(\mathrm{kJ} \mathrm{mol}^{-1} \mathrm{~K}^{-1}\right)\end{array}$} & \multicolumn{6}{|c|}{$\mathrm{y}=\mathrm{A} e^{-k t}$} \\
\hline & & & & & & & \multirow[b]{2}{*}{$\begin{array}{l}\mathrm{R}^{2} \text { of } \\
\text { curve } \\
\text { fitting }\end{array}$} & \multirow[b]{2}{*}{ A } & \multicolumn{2}{|c|}{ Agg kinetic constant $k$} & \multicolumn{2}{|c|}{ Initial agg rate $v=A k$} \\
\hline & & & & & & & & & $\left(\min ^{-1}\right)$ & $\begin{array}{c}k \\
\text { improvement } \\
\text { to WT }\end{array}$ & $\begin{array}{c}\ln (v) \\
\left(\% \text { day }^{-1}\right)\end{array}$ & $\begin{array}{c}v \\
\text { improvement } \\
\text { to WT }\end{array}$ \\
\hline & Wild type & 71.80 & 63.06 & $4.74 \%$ & 429.10 & 1.24 & 0.9976 & 0.9700 & 0.042 & $\mathrm{~N} / \mathrm{A}$ & 8.6656 & \begin{tabular}{|l|}
$\mathrm{N} / \mathrm{A}$ \\
\end{tabular} \\
\hline \multirow{12}{*}{ Stable } & HC-A227E & 71.23 & 63.20 & $4.74 \%$ & 467.15 & 1.36 & 0.9981 & 0.9465 & 0.043 & $-2.72 \%$ & 8.6679 & $-0.23 \%$ \\
\hline & $\mathrm{HC}-\mathrm{A} 228 \mathrm{H}$ & 70.70 & 62.78 & $5.86 \%$ & 471.98 & 1.37 & 0.9992 & 0.9602 & 0.039 & $5.56 \%$ & 8.5982 & $6.51 \%$ \\
\hline & HC-T135Y & 72.13 & 63.79 & $3.50 \%$ & 451.48 & 1.31 & 0.9984 & 0.9888 & 0.040 & $4.50 \%$ & 8.6387 & $2.65 \%$ \\
\hline & HC-S134Y & 72.21 & 63.46 & $3.95 \%$ & 429.92 & 1.24 & 0.9986 & 0.9821 & 0.042 & $-1.88 \%$ & 8.6965 & $-3.15 \%$ \\
\hline & HC-S134M & 72.46 & 63.49 & $3.84 \%$ & 419.60 & 1.21 & 0.9992 & 0.9595 & 0.040 & $2.87 \%$ & 8.6255 & $3.92 \%$ \\
\hline & HC-S134P & 72.30 & 63.44 & $3.95 \%$ & 424.75 & 1.23 & 0.9985 & 0.9582 & 0.044 & $-5.61 \%$ & 8.7078 & $-4.33 \%$ \\
\hline & HC-S136G & 72.09 & 63.37 & $4.12 \%$ & 430.94 & 1.25 & 0.9991 & 0.9421 & 0.043 & $-3.68 \%$ & 8.6725 & $-0.71 \%$ \\
\hline & HC-S219Y & 70.83 & 63.05 & $5.42 \%$ & 481.17 & 1.40 & 0.9994 & 0.9541 & 0.037 & $11.25 \%$ & 8.5296 & $12.70 \%$ \\
\hline & LC-L154A & 72.05 & 63.65 & $3.72 \%$ & 448.13 & 1.30 & 0.9983 & 0.9659 & 0.042 & $-1.32 \%$ & 8.6744 & $-0.90 \%$ \\
\hline & LC-S176W & $\mathrm{N} / \mathrm{A}$ & $\mathrm{N} / \mathrm{A}$ & $\mathrm{N} / \mathrm{A}$ & $\mathrm{N} / \mathrm{A}$ & $\mathrm{N} / \mathrm{A}$ & 0.9980 & 0.9552 & 0.060 & $-44.75 \%$ & 9.0201 & $-42.55 \%$ \\
\hline & HC-G194H & 70.91 & 63.43 & $4.49 \%$ & 500.69 & 1.46 & 0.9990 & 0.9561 & 0.037 & $11.34 \%$ & 8.5307 & $12.62 \%$ \\
\hline & HC-T197L & 70.19 & 60.83 & $10.64 \%$ & 396.65 & 1.16 & 0.9968 & 0.9558 & 0.051 & $-23.12 \%$ & 8.8587 & $-21.32 \%$ \\
\hline \multirow{5}{*}{ Unstable } & LC-A153P & 67.13 & 59.57 & $25.37 \%$ & 486.92 & 1.43 & 0.9899 & 0.9893 & 0.179 & $-330.23 \%$ & 10.1443 & $-338.79 \%$ \\
\hline & LC-G66P & 60.17 & 50.35 & $86.17 \%$ & 355.29 & 1.07 & 0.9944 & 0.8694 & 1.648 & $-3869.29 \%$ & 12.2372 & $-3457.62 \%$ \\
\hline & LC-G200W & 66.25 & 58.39 & $35.25 \%$ & 463.73 & 1.37 & 0.9826 & 1.0033 & 0.267 & $-543.69 \%$ & 10.5613 & $-565.79 \%$ \\
\hline & HC-V215W & 66.18 & 58.92 & $34.91 \%$ & 502.17 & 1.48 & 0.9875 & 1.0288 & 0.203 & $-389.55 \%$ & 10.3127 & $-419.21 \%$ \\
\hline & HC-G178P & 63.89 & 55.57 & $62.41 \%$ & 431.99 & 1.28 & 0.9721 & 1.0351 & 0.285 & $-586.61 \%$ & 10.6571 & $-632.70 \%$ \\
\hline
\end{tabular}

Summary of values obtained for the thermal stability measurements and aggregation kinetics, for all the variants tested.

\section{Correlations Between Aggregation Rate and Thermal Stability Parameters}

Correlations between the aggregation rates, $\ln (v)$, and both $T_{\mathrm{m}}$ and $T_{\mathrm{on}}$ are shown in

Figure 6(A). As expected, inverse correlations were found, where a decrease in $T_{\mathrm{m}}$

and $T_{\mathrm{on}}$ resulted in more monomer loss due to conformational instability. Both $T_{\mathrm{m}}$ and $T_{\text {on }}$ correlated well $\left(\mathrm{R}^{2}=0.95\right)$ with the aggregation rate, with the correlations mainly driven by the inclusion of the five destabilising variants that were considerably unfolded at $65^{\circ} \mathrm{C}$. There was indeed no correlation found between aggregation rates and $T_{\mathrm{m}}$, when comparing the stable variants alone, consistent with a different aggregation mechanism to that of the unstable variants (SI). The initial aggregation rates were also plotted against the fraction of unfolded state at $65^{\circ} \mathrm{C}$, at which the kinetic study was operated (Figure 6(B)). Although a good linear correlation $\left(\mathrm{R}^{2}=0.94\right)$ was obtained between aggregation rate and $f_{\mathrm{T} 65}$ when combining all the 
variants, it could be seen that when the fraction of unfolded state was below $6 \%$, the aggregation rate was essentially constant, and deviated from the linear fit. Various reaction models and their fits to the data are discussed further below.

As described above, HC-A228H, HC-S219Y and HC-G194H reduced the aggregation rate the most, by $6-12 \%$, whereas their $T_{\mathrm{m}}$ values were even one degree lower than that of wild type. This implied that they could be in, or close to, particular hotspots of sequence or structure that affect the aggregation mechanism, and yet the one-degree reduction in $T_{\mathrm{m}}$ had little influence on local dynamics. To visualise this, the locations of all stable variants with $\ln (v) 8.5$ to $8.8 \%$ day $^{-1}$ were highlighted in Figure 6(C). It shows that the three top mutations were located close together at the Cterminal end of the heavy chain. Two of them, HC-A228 and HC-G194 were mutated to Histidine. These residues were $5.0 \AA$ and $7.3 \AA$ from potential salt-bridge partners HC-D221 and HC-E216, respectively in the energy minimised model from Gromacs, which could provide a route to pin the flexible terminal chain into place. Another reason might be the additional contacts provided by the mutation. As shown in the SI, compared to the wild type at the mutational site, HC-A228 and HC-S219Y variants created respectively three and one new contacts, while HC-G194H replaced a hydrogen bond to HC-Ser191 with a van der Waals interaction to HC-Pro217. These changes upon mutations each increase the number of non-covalent interactions to the hinge region. As a result, these three variants reduced the local loop flexibility, which also led to the increased unfolding cooperativity as shown by their decrease in $S_{\mathrm{N}}$ (increased $\left.\Delta S_{\mathrm{vh}}\right)$ compared to the wild type. 
Variant HC-T197L was also near to the heavy-chain C-terminus, decreased the $T_{\mathrm{m}}$ by $1.6^{\circ} \mathrm{C}$, and showed a $21 \%$ increase in the aggregation rate. Considering the decrease in $\Delta S_{\mathrm{vh}}$ for HC-T197L (increased $S_{\mathrm{N}}$ ) of $1.16 \mathrm{~kJ} \mathrm{~mol}^{-1} \mathrm{~K}^{-1}$, compared to $1.24 \mathrm{~kJ} \mathrm{~mol}^{-1}$ $\mathrm{K}^{-1}$ for wild type, this mutation appeared to have induced greater local conformational flexibility and significant loss of unfolding cooperativity.

Among the stable variants, five targeted the flexible loop regions at heavy chain residues 134-136. However, they did not alter the $T_{\mathrm{m}}$ or the unfolding cooperativities, and they all had similar aggregation rates to the wild type (within 5\%). Rosetta predicts these mutations to be more stable potentially through local hydrophobic interactions. The SI shows that the two Tyr variants, HC-T135Y and HC-S134Y added two more van der Waals contacts while the other three variants remained the same as the wild type. Considering their comparable aggregation rate, any improved conformational stability, particularly for $\mathrm{T} 135 \mathrm{Y}$, might be compensated by their increased propensity for hydrophobic interaction with other proteins. Indeed, four out of five mutations on HC-134-136 had an increased hydrophobic fraction of solvent accessible surface area (SASA) $)^{59,60}$ (SI). However, HC-S219Y and HC-G194H also had higher hydrophobic SASA than the wild type, and yet lower aggregation rates, which implies that the relatively modest changes in hydrophobicity from such mutations are not the main factor affecting the aggregation rate. More likely, the flexible loop region $\mathrm{HC}-134-136$ was not involved in the aggregation mechanism to the same extent as the C-terminus, although there remained scope to explore this further given the relatively small changes in cooperativity and thermal stability for mutations 
in this region. Another stable variant LC-L154A reduced one contact but also had less hydrophobic SASA, yet had a very similar aggregation rate to the wild type. Further mutations in that light chain loop 151-157 would be needed to fully determine its role in aggregation from the native ensemble. In conclusion, the aggregation rate was influenced by the flexibility of specific surface loops, but not for all loops.

A

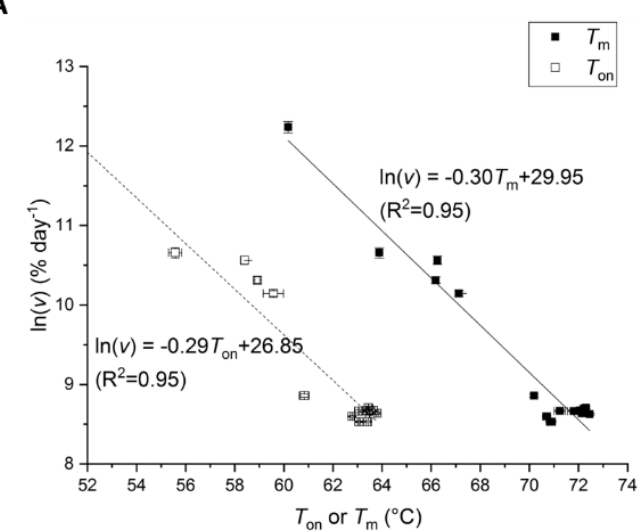

C

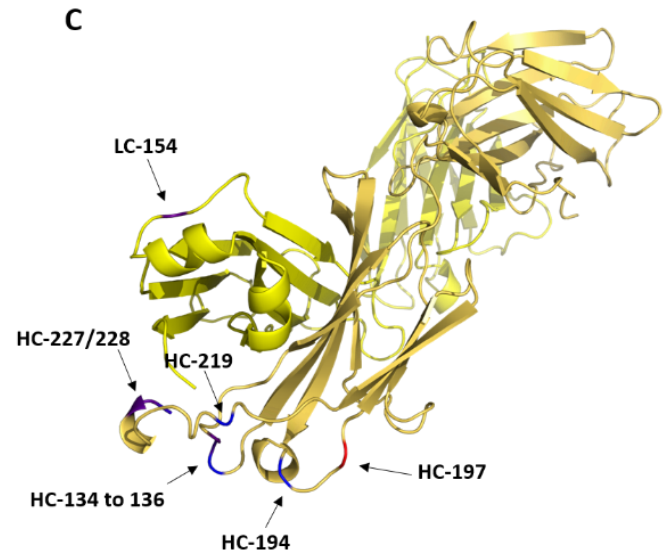

B

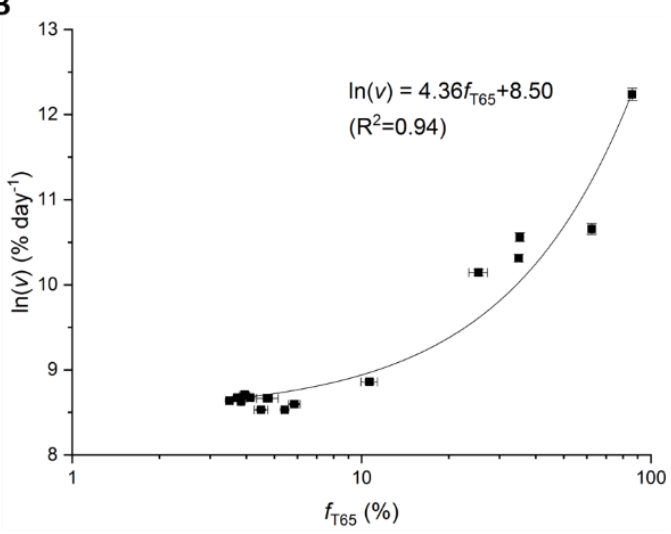

Figure 6 The correlations between initial aggregation rate and thermal stability (LCS176W excluded), and the locations of stable variants coloured by their aggregation rate

(A) Linear correlations between initial aggregation rate, and both $T_{\mathrm{on}}$, and $T_{\mathrm{m}}$. 
(B) Linear correlation between initial aggregation rates and $f_{\text {T65 }}$ (plotted on $\log _{10}$ scale)

(C) The locations of stable variants with aggregation rate $\ln (\mathrm{v}) 8.53$ to $8.86 \%$ day-1 were coloured in gradient from blue to red, indicated by arrows. The light chain and heavy chain were coloured in yellow and orange, respectively.

We fit the data in Figure 7B to a range of potential kinetic models as previously for $\mathrm{GCSF}^{54}$, assuming that all variants underwent the same aggregation mechanism, but where aggregation could occur from both a native-like species or a globally unfolded species, with relative rates that depend upon the fraction unfolded, and hence the global unfolding stability of each variant. Two numerical models gave good fits. The first $\left(\mathrm{R}^{2}=0.96\right)$ was a bimolecular diffusion-limited aggregation from both $\mathrm{N}^{*}$ and $\mathrm{U}^{*}$, which are in rapid pre-equilibria with $\mathrm{N}$ and $\mathrm{U}$, respectively. The second $\left(\mathrm{R}^{2}=0.96\right)$, was the numerical solution to at least three reaction models (3a, $3 b$, and $3 c$ in SI). Model 3a was for a monomolecular reaction from $\mathrm{N}$, combined with a bimolecular diffusion-limited reaction from $U^{*}$. Model $3 b$ was for a monomolecular reaction from $\mathrm{N}^{*}$ in rapid pre-equilibrium with $\mathrm{N}$, combined with a bimolecular diffusion-limited reaction from $\mathrm{U}^{*}$. Model $3 \mathrm{c}$ was for aggregation from $\mathrm{N}^{*}$, formed in a rate-limiting monomolecular reaction from $\mathrm{N}$, combined with a bimolecular diffusion-limited reaction from $\mathrm{U}^{*}$.

As the rate order was determined to be $0.59-1$, models $3 a-c$ were more likely than model 1 , as it included a component that was monomolecular from $\mathrm{N}$ or $\mathrm{N}^{*}$. Of these 
three models, $3 \mathrm{c}$ the rate-limiting monomolecular reaction from $\mathrm{N}$ to $\mathrm{N}^{*}$ could have a physical basis in a relatively slow partial-unfolding step. Accordingly, at lower protein concentrations than that studied, the subsequent aggregation step might be expected to become rate-limiting instead. By contrast, $3 \mathrm{a}$ and $3 \mathrm{~b}$ involve a monomolecular aggregate-forming step, which could have a physical basis in the reaction between $\mathrm{N}$ or $\mathrm{N}^{*}$ and pre-existing aggregate nuclei or fragments. However, this would also require that the concentration of binding sites for $\mathrm{N}$ or $\mathrm{N}^{*}$ within the nuclei, does not increase over time.

For model 3c, the kinetic constant for partial unfolding of $\mathrm{N}^{*} \mathrm{~N}^{*}, k_{4}=6.4 \pm 0.5 \times 10^{-}$ ${ }^{4} \mathrm{~s}^{-1}$. Variants that affect only the global unfolding stability would not necessarily affect this rate constant. However, variants that deviate from the fit to model $3 \mathrm{c}$ in SI, are potentially those that modify $k_{4}$, through direct influence on the partially unfolding regions of structure, such as those in the heavy-chain C-terminus as discussed above. The equilibrium constant $K_{\mathrm{U}^{*}}=\left[\mathrm{U}^{*}\right] /[\mathrm{U}]=2.6 \pm 0.1 \times 10^{-4}$, indicated a population of $\mathrm{U}^{*}$ that was one part in 3846 of the unfolded population. Alternative models would also fit the data for the unfolded population, including one in which aggregation occurs directly from diffusion-limited collisions between two unfolded molecules $U$, but where formation of aggregate depends on molecular orientation as described by a transmission coefficient $\tau$, numerically equivalent to $K_{\mathrm{U}^{*}}{ }^{2}$, hence $\tau=6.8 \times 10^{-8}$. However, for the most stable variants, where the protein is predominantly native, the aggregation mechanism is essentially only monomolecular from $\mathrm{N}$, without global unfolding to $\mathrm{U}$. 


\section{Are Point Mutations Sufficient to Stabilise The Fab?}

Previously, the wild-type A33 Fab was examined for its aggregation rate across a wide range of $\mathrm{pH}$, ionic strength and temperatures ${ }^{36}$. Our current work explored 17 point-variants, but in only one formulation condition of $\mathrm{pH} \mathrm{4,} 200 \mathrm{mM}$ ionic strength at $65^{\circ} \mathrm{C}$. At $\mathrm{pH} 4.5,200 \mathrm{mM}$ ionic strength previously, the Fab did not follow a linear Arrhenius behaviour between aggregation rate and the reciprocal of temperature. Instead, the unfolded fraction substantially increased from $<0.00004 \%$ at $45{ }^{\circ} \mathrm{C}$ and below, to approximately $2 \%$ at $65^{\circ} \mathrm{C}$, and contributed more towards the aggregation rate, as discussed before.

In Figure 7, the wild type data from $\mathrm{pH} 3.5-9,65^{\circ} \mathrm{C}^{36}$ was plotted along with our current variant data (from Figure 6B). A distinct difference is clearly shown for data at the different $\mathrm{pH}$, except for conditions with very low ionic strength, where colloidal stabilisation through intermolecular electrostatic repulsion is presumably more significant. For a particular $\mathrm{pH}$, the $\mathrm{Fab}$ aggregation rate data deviated over a relatively small range, due to their difference in ionic strength or applied mutations. This information suggests that, for the $\mathrm{A} 33 \mathrm{Fab}$, the optimisation of $\mathrm{pH}$ should take priority above that of ionic strength, or single point mutations.

Figure 7 also shows a steady increase of $\ln (v)$ from 0 to 3 as $f_{\mathrm{T} 65}$ increases from less than 0.001 to $2 \%$ (mostly $\mathrm{pH} 5.5-9)$, followed by a sharp increase of $\ln (v)$ from 5 to 12 as $f_{\mathrm{T} 65}$ increases from 0.5 to nearly $100 \%$ (mostly $\mathrm{pH} 3.5-4.5$ ). This suggests that, when the unfolded states accounted mainly for the protein aggregation, the aggregation could be easily minimised through the optimisation of $T_{\mathrm{m}}$ (e.g. by mutation). 
However, when the protein is already within, or close to, the baseline aggregation rate under native conditions, little improvement could be achieved by reducing the unfolded fraction. It has been found ${ }^{54}$ that GCSF has a baseline rate of aggregation 66-fold higher than the A33 Fab. Thus, this baseline rate relates to the unique feature of a certain native protein. To further reduce this baseline, multiple sites of the protein would need to be modified to alter its local dynamics, if its efficacy is not compromised.

One additional feature observed in the present work, is that a separate baseline is reached within the $\mathrm{pH} 4$ data for the variants. This indicates that the native-like state from which aggregation occurs in these conditions, is itself $\mathrm{pH}$ dependent.

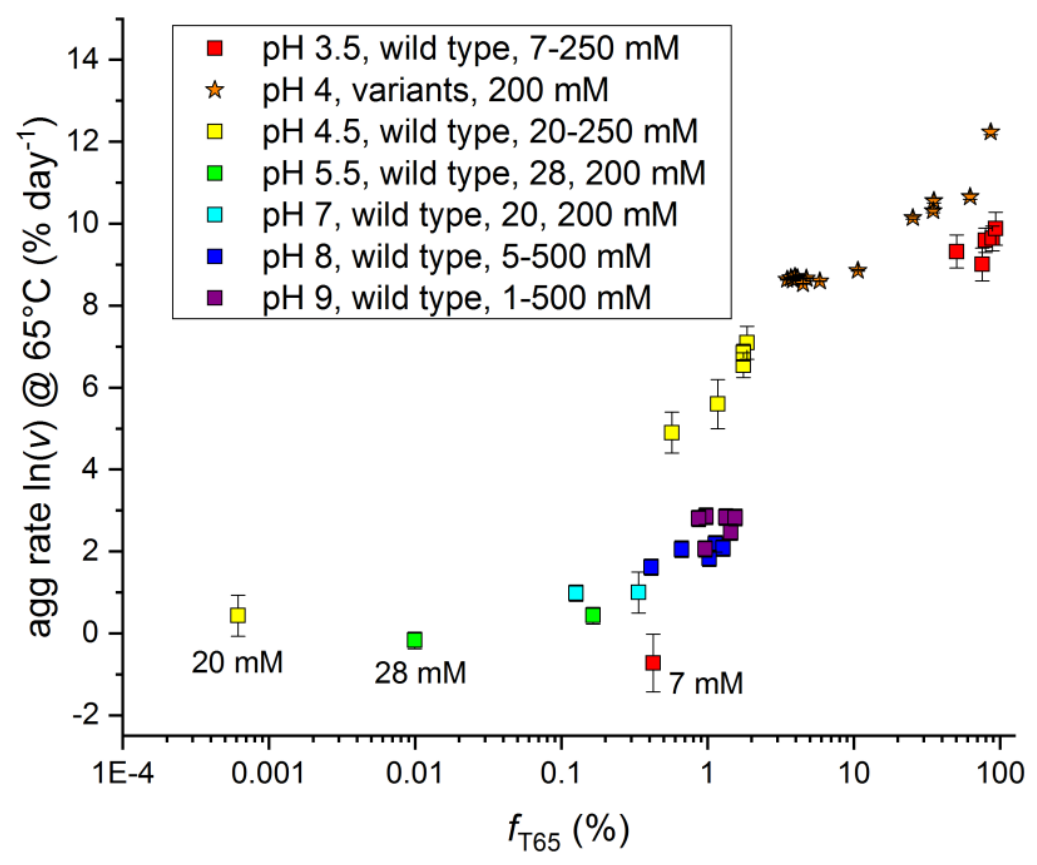

Figure 7 The aggregation rate and the unfolded fraction at $65^{\circ} \mathrm{C}$

Data from different $\mathrm{pH}$ conditions was coloured individually. The wild type data was derived from Chakroun et al. 2016, and was indicated by squares; the variants data from this work was indicated by stars. 


\section{CONCLUSION}

This work used multiple protein modelling tools to explore the effect of point mutations on the protein global stability, unfolding cooperativity as a proxy for native conformational flexibility, and aggregation rates under conditions at which wild-type is only $4.7 \%$ unfolded. The protein was known from previous work to be very stable to global unfolding. Consistent with this, the predictions from Rosetta across all residues in the protein suggested only modest $(<1 \%)$ improvements in stability would be possible from single mutations. However, this provided an opportunity to target the conformational flexibility of the native protein, and measure the impact of this on unfolding cooperativity and also on aggregation under predominantly native conditions. None of the stabilising mutations targeted to flexible regions had significant impact on the $T_{\mathrm{m}}$-values of the A33 Fab, as expected. However, six variants increased $\Delta S_{\mathrm{vh}}$, indicating increased unfolding cooperativity consistent with fewer conformations in the native state ensemble. Of these, three variants reduced the aggregation rate by $6-12 \%$, compared to the wild type, and were co-located around the C-terminal end of the heavy chain. Other variants revealed increased unfolding cooperativities through stabilisation of the loop regions of residue 154 in the light chain, and residue 135 in the heavy chain, but without reducing the aggregation rate compared to the wild type. Thus, while not all flexible loops were important for Fab aggregation, the local dynamics of the heavy-chain of the $\mathrm{C}$-terminus played a role in the aggregation mechanism. It will be interesting in future to target multiple mutations to each flexible 
region, to identify additive or synergistic effects on the unfolding cooperativity and aggregation kinetics.

Fitting of a series of alternative kinetic models to the dependence on fraction unfolded for the variants, revealed that aggregation most likely occurred via a ratelimiting partial-unfolding step under predominantly native conditions. This model is consistent with the observation that rigidifying certain surface loops could decrease the rates of monomer loss.

\section{ASSOCIATED CONTENT}

Supporting Information

The procedure of homology modelling; correlations between RMSF and B-factor broken down by secondary structure; in silico single-point mutagenesis for $\Delta \Delta G$; homologous structures used for B-factor alignment; the primers of the designed variants; the thermal stability data and aggregation kinetics for the variants; correlations between aggregation rate and $T_{\mathrm{m}}, T_{\mathrm{on}}$ and $f_{\mathrm{T} 65}$ for stable variants only; kinetics of monomer loss at various initial concentrations of wild-type Fab; the unique features of LC-S176W variant; the non-covalent contacts change at the mutational points; the solvent accessible surface area (SASA) at the mutational points.

\section{ACKNOWLEDGEMENT}

The support of the Engineering and Physical Sciences Research Council (EPSRC)

Centre for Innovative Manufacturing in Emergent Macromolecular Therapies (EP/I033270/1), and the EPSRC Future Targeted Healthcare Manufacturing Hub (EP/P006485/1) is gratefully acknowledged. The Hub is part of the Advanced Centre for Biochemical Engineering, Department of Biochemical Engineering, University College London. The authors thank UCB Pharma for providing the A33 Fab. The 
authors acknowledge the use of the UCL Legion High-Performance Computing Facility (Legion@UCL), and associated support services, in the completion of this work.

\section{REFERENCE}

(1) Roberts, C. J. Protein Aggregation and Its Impact on Product Quality. Curr. Opin. Biotechnol. 2014, 30, 211-217.

(2) Vázquez-Rey, M.; Lang, D. A. Aggregates in Monoclonal Antibody Manufacturing Processes. Biotechnol. Bioeng. 2011, 108 (7), 1494-1508.

(3) Samra, H. S.; He, F. Advancements in High Throughput Biophysical Technologies: Applications for Characterization and Screening during Early Formulation Development of Monoclonal Antibodies. Mol. Pharm. 2012, 9(4), 696-707.

(4) Mueller, M.; Loh, M. Q. T.; Tee, D. H. Y.; Yang, Y.; Jungbauer, A. Liquid Formulations for Long-Term Storage of Monoclonal IgGs. Appl. Biochem. Biotechnol. 2013, 169 (4), $1431-1448$.

(5) Neergaard, M. S.; Nielsen, A. D.; Parshad, H.; De Weert, M. Van. Stability of Monoclonal Antibodies at High-Concentration: Head-to-Head Comparison of the IgG1 and IgG4 Subclass. J. Pharm. Sci. 2014, $103(1), 115-127$.

(6) Jezek, J.; Rides, M.; Derham, B.; Moore, J.; Cerasoli, E.; Simler, R.; Perez-Ramirez, B. Viscosity of Concentrated Therapeutic Protein Compositions. Adv. Drug Deliv. Rev. 2011, $63(13), 1107-1117$ 
(7) Ratanji, K. D.; Derrick, J. P.; Dearman, R. J.; Kimber, I. Immunogenicity of Therapeutic Proteins: Influence of Aggregation. J. Immunotoxicol. 2013, 11 (2), 99-109.

(8) Moussa, E. M.; Panchal, J. P.; Moorthy, B. S.; Blum, J. S.; Joubert, M. K.; Narhi, L. O.; Topp, E. M. Immunogenicity of Therapeutic Protein Aggregates. J. Pharm. Sci. 2016, $105(2), 417-430$.

(9) van der Kant, R.; Karow-Zwick, A. R.; Van Durme, J.; Blech, M.; Gallardo, R.; Seeliger, D.; Aßfalg, K.; Baatsen, P.; Compernolle, G.; Gils, A.; et al. Prediction and Reduction of the Aggregation of Monoclonal Antibodies. J. Mol. Biol. 2017, 429 (8), 1244-1261.

(10) Lauer, T. M.; Agrawal, N. J.; Chennamsetty, N.; Egodage, K.; Helk, B.; Trout, B. L. Developability Index: A Rapid in Silico Tool for the Screening of Antibody Aggregation Propensity. J. Pharm. Sci. 2012, 101 (1), 102-115.

(11) Wijma, H. J.; Floor, R. J.; Janssen, D. B. Structure- and Sequence-Analysis Inspired Engineering of Proteins for Enhanced Thermostability. Curr. Opin. Struct. Biol. 2013, 23 (4), 588-594.

(12) Brooks, B. R.; Brooks, C. L.; MacKerell, A. D.; Nilsson, L.; Petrella, R. J.; Roux, B.; Won, Y.; Archontis, G.; Bartels, C.; Boresch, S.; et al. CHARMM: The Biomolecular Simulation Program. J. Comput. Chem. 2009, 30(10), 1545-1614.

(13) Pronk, S.; Páll, S.; Schulz, R.; Larsson, P.; Bjelkmar, P.; Apostolov, R.; Shirts, M. R.; Smith, J. C.; Kasson, P. M.; Van Der Spoel, D.; et al. GROMACS 4.5: A HighThroughput and Highly Parallel Open Source Molecular Simulation Toolkit. 
Bioinformatics 2013, 29 (7), 845-854.

(14) Abraham, M. J.; Murtola, T.; Schulz, R.; Páall, S.; Smith, J. C.; Hess, B.; Lindah, E. Gromacs: High Performance Molecular Simulations through Multi-Level Parallelism from Laptops to Supercomputers. SoftwareX 2015, 1-2, 19-25.

(15) Case, D. A.; Cheatham, T. E.; Darden, T.; Gohlke, H.; Luo, R.; Merz, K. M.; Onufriev, A.; Simmerling, C.; Wang, B.; Woods, R. J. The Amber Biomolecular Simulation Programs. J. Comput. Chem. 2005, 26 (16), 1668-1688.

(16) Salomon-Ferrer, R.; Case, D. A.; Walker, R. C. An Overview of the Amber Biomolecular Simulation Package. Wiley Interdiscip. Rev. Comput. Mol. Sci. 2013, 3(2), 198-210.

(17) Ohmura, T.; Ueda, T.; Ootsuka, K.; Saito, M.; Imoto, T. Stabilization of Hen Egg White Lysozyme by a Cavity-Filling Mutation. Protein Sci. 2001, 10 (2), 313-320.

(18) van der Kamp, M. W.; Daggett, V. Pathogenic Mutations in the Hydrophobic Core of the Human Prion Protein Can Promote Structural Instability and Misfolding. J. Mol. Biol. 2010, 404 (4), 732-748.

(19) Yu, H.; Huang, H. Engineering Proteins for Thermostability through Rigidifying Flexible Sites. Biotechnol. Adv. 2014, 32 (2), 308-315.

(20) Yuan, Z.; Bailey, T. L.; Teasdale, R. D. Prediction of Protein B-Factor Profiles. Proteins Struct. Funct. Genet. 2005, 58 (4), 905-912.

(21) Reetz, M. T.; Carballeira, J. D. Iterative Saturation Mutagenesis (ISM) for Rapid Directed Evolution of Functional Enzymes. Nat. Protoc. 2007, 2 (4), 891-903. 
(22) Yu, H.; Yan, Y.; Zhang, C.; Dalby, P. A. Two Strategies to Engineer Flexible Loops for Improved Enzyme Thermostability. Sci. Rep. 2017, 7 (December 2016), 41212.

(23) Potapov, V.; Cohen, M.; Schreiber, G. Assessing Computational Methods for Predicting Protein Stability upon Mutation: Good on Average but Not in the Details. Protein Eng. Des. Sel. 2009, 22 (9), 553-560.

(24) Khan, S.; Vihinen, M. Performance of Protein Stability Predictors. Hum. Mutat. 2010, 31 (6), 675-684.

(25) Niroula, A.; Vihinen, M. Variation Interpretation Predictors: Principles, Types, Performance and Choice. Hum. Mutat. 2016.

(26) Chivian, D.; Baker, D. Homology Modeling Using Parametric Alignment Ensemble Generation with Consensus and Energy-Based Model Selection. Nucleic Acids Res. 2006, $34(17), \mathrm{e} 112$.

(27) Kuhlman, B.; Dantas, G.; Ireton, G. Design of a Novel Globular Protein Fold with AtomicLevel Accuracy. Science. 2003, 302, 1364-1368.

(28) Leaver-Fay, A.; Tyka, M.; Lewis, S. M.; Lange, O. F.; Thompson, J.; Jacak, R.; Kaufman, K.; Renfrew, P. D.; Smith, C. A.; Sheffler, W. R 3: An Object-Oriented Software Suite for the Simulation and Design of Macromolecules. Methods Enzymol 2014, 487, 545574.

(29) Kellogg, E. H.; Leaver-Fay, A.; Baker, D. Role of Conformational Sampling in Computing Mutation-Induced Changes in Protein Structure and Stability. Proteins Struct. Funct. 
Bioinforma. 2011, 79 (3), 830-838.

(30) Costanzo, J. A.; O’Brien, C. J.; Tiller, K.; Tamargo, E.; Robinson, A. S.; Roberts, C. J.; Fernandez, E. J. Conformational Stability as a Design Target to Control Protein Aggregation. Protein Eng. Des. Sel. 2014, $27(5), 157-167$.

(31) Chiu, M. L.; Gilliland, G. L. Engineering Antibody Therapeutics. Curr. Opin. Struct. Biol. 2016, 38, 163-173.

(32) Shukla, A. A.; Thömmes, J. Recent Advances in Large-Scale Production of Monoclonal Antibodies and Related Proteins. Trends Biotechnol. 2010, 28 (5), 253-261.

(33) Mitragotri, S.; Burke, P. A.; Langer, R. Overcoming the Challenges in Administering Biopharmaceuticals: Formulation and Delivery Strategies. Nat. Rev. Drug Discov. 2014, $13(9), 655-672$.

(34) Nelson, A. L. Antibody Fragments: Hope and Hype. Landes Biosci. 2010, 2 (1), 77-83.

(35) Holliger, P.; Hudson, P. J. Engineered Antibody Fragments and the Rise of Single Domains. Nat. Biotechnol. 2005, 23 (9), 1126-1136.

(36) Chakroun, N.; Hilton, D.; Ahmad, S. S.; Platt, G. W.; Dalby, P. A. Mapping the Aggregation Kinetics of a Therapeutic Antibody Fragment. Mol. Pharm. 2016, 13 (2), 307-319.

(37) Brader, M. L.; Estey, T.; Bai, S.; Alston, R. W.; Lucas, K. K.; Lantz, S.; Landsman, P.; Maloney, K. M. Examination of Thermal Unfolding and Aggregation Profiles of a Series of Developable Therapeutic Monoclonal Antibodies. Mol. Pharm. 2015, 12 (4), 1005- 
1017.

(38) Uchiyama, S. Liquid Formulation for Antibody Drugs. Biochim. Biophys. Acta - Proteins Proteomics 2014, 1844 (11), 2041-2052.

(39) Dolinsky, T. J.; Nielsen, J. E.; McCammon, J. A.; Baker, N. A. PDB2PQR: An Automated Pipeline for the Setup of Poisson-Boltzmann Electrostatics Calculations. Nucleic Acids Res. 2004, 32 (WEB SERVER ISS.), 665-667.

(40) Li, H.; Robertson, A. D.; Jensen, J. H. Very Fast Empirical Prediction and Rationalization of Protein pK a Values. Proteins Struct. Funct. Genet. 2005, 61 (4), 704-721.

(41) Rose, P. W.; Bi, C.; Bluhm, W. F.; Christie, C. H.; Dimitropoulos, D.; Dutta, S.; Green, R. K.; Goodsell, D. S.; Prli??, A.; Quesada, M.; et al. The RCSB Protein Data Bank: New Resources for Research and Education. Nucleic Acids Res. 2013, 41 (D1), 475482.

(42) Hall, T. BioEdit: A User-Friendly Biological Sequence Alignment Editor and Analysis Program for Windows 95/98/NT. Nucleic Acids Symposium Series. 1999, pp 95-98.

(43) Kellogg, E. H.; Leaver-Fay, A.; Baker, D. Role of Conformational Sampling in Computing Mutation-Induced Changes in Protein Structure and Stability. Proteins Struct. Funct. Bioinforma. 2011, 79(3), 830-838.

(44) Santoro, M. M.; Bolen, D. W. Unfolding Free Energy Changes Determined by the Linear Extrapolation Method. 1. Unfolding of Phenylmethanesulfonyl a-Chymotrypsin Using Different Denaturants. Biochemistry 1988, 27(21), 8063-8068. 
(45) Consalvi, V.; Chiaraluce, R.; Giangiacomo, L.; Scandurra, R.; Christova, P.; Karshikoff, A.; Knapp, S.; Ladenstein, R. Thermal Unfolding and Conformational Stability of the Recombinant Domain II of Glutamate Dehydrogenase from the Hyperthermophile Thermotoga Maritima. Protein Eng. 2000, 13 (7), 501-507.

(46) Studer, R. A.; Dessailly, B. H.; Orengo, C. A. Residue Mutations and Their Impact on Protein Structure and Function: Detecting Beneficial and Pathogenic Changes. Biochem. J. 2013, 449(3), 581-594.

(47) Nick Pace, C.; Martin Scholtz, J.; Grimsley, G. R. Forces Stabilizing Proteins. FEBS Lett. 2014, $588(14), 2177-2184$.

(48) Teilum, K.; Olsen, J. G.; Kragelund, B. B. Protein Stability, Flexibility and Function. Biochim. Biophys. Acta - Proteins Proteomics 2011, 1814 (8), 969-976.

(49) Lee, S.; Antony, L.; Hartmann, R.; Knaus, K. J.; Surewicz, K.; Surewicz, W. K.; Yee, V. C. Conformational Diversity in Prion Protein Variants Influences Intermolecular BetaSheet Formation. EMBO J. 2010, 29 (1), 251-262.

(50) Dupuis, N. F.; Wu, C.; Shea, J. E.; Bowers, M. T. The Amyloid Formation Mechanism in Human IAPP: Dimers Have Beta-Strand Monomer-Monomer Interfaces. J Am Chem Soc 2011, $133(19), 7240-7243$.

(51) Zambrano, R.; Jamroz, M.; Szczasiuk, A.; Pujols, J.; Kmiecik, S.; Ventura, S. AGGRESCAN3D (A3D): Server for Prediction of Aggregation Properties of Protein Structures. Nucleic Acids Res. 2015, 43(W1), W306-W313. 
(52) King, D. J.; Antoniw, P.; Owens, R. J.; Adair, J. R.; Mountain, A.; Farnsworth, A. P. H.; Finney, H.; Lawson, A. D. G.; Lyons, A.; Baker, T. S.; et al. Preparation and Preclinical Evaluation of Humanised A33 Immunoconjugates for Radioimmunotherapy. Br. J. Cancer 1995, $72(6)$, 1364-1372.

(53) Krishnan, S.; Chi, E. Y.; Webb, J. N.; Chang, B. S.; Shan, D.; Goldenberg, M.; Manning, M. C.; Randolph, T. W.; Carpenter, J. F. Aggregation of Granulocyte Colony Stimulating Factor under Physiological Conditions: Characterization and Thermodynamic Inhibition. Biochemistry 2002, 41 (20), 6422-6431.

(54) Robinson, M. J.; Matejtschuk, P.; Bristow, A. F.; Dalby, P. A. Tm-Values and Unfolded Fraction Can Predict Aggregation Rates for GCSF Variant Formulations, but Not under Predominantly Native Conditions. Mol. Pharm. 2017.

(55) Roberts, C. J. Non-Native Protein Aggregation Kinetics. Biotechnol. Bioeng. 2007, 98 (5), 927-938.

(56) Shakhnovich, E. I.; Finkelstein, A. V. Theory of Cooperative Transitions in Protein Molecules. I. Why Denaturation of Globular Protein Is a First-order Phase Transition. Biopolymers 1989, $28(10)$, 1667-1680.

(57) Dagan, S.; Hagai, T.; Gavrilov, Y.; Kapon, R.; Levy, Y.; Reich, Z. Stabilization of a Protein Conferred by an Increase in Folded State Entropy. Proc. Natl. Acad. Sci. 2013, $110(26), 10628-10633$.

(58) Wang, W.; Nema, S.; Teagarden, D. Protein Aggregation--Pathways and Influencing 
Factors. Int. J. Pharm. 2010, 390 (2), 89-99.

(59) Fraternali, F.; Cavallo, L. Parameter Optimized Surfaces (POPS): Analysis of Key Interactions and Conformational Changes in the Ribosome. Nucleic Acids Res. 2002, 30 (13), 2950-2960.

(60) Cavallo, L.; Kleinjung, J.; Fraternali, F. POPS: A Fast Algorithm for Solvent Accessible Surface Areas at Atomic and Residue Level. Nucleic Acids Res. 2003, 31 (13), 33643366. 